\title{
EFFECT OF GAMMA IRRADIATION AND SOME HOLDING SOLUTIONS ON VASE LIFE OF GERBERA CUT FLOWERS
}

\author{
Ola A. Amin; Azza M. Abdel-Moneim and Hanan M.A.Youssef \\ Ornamental Plants and Landscape Gardening Res. Dept., Hort. Res. Inst., ARC, Giza, Egypt.
}

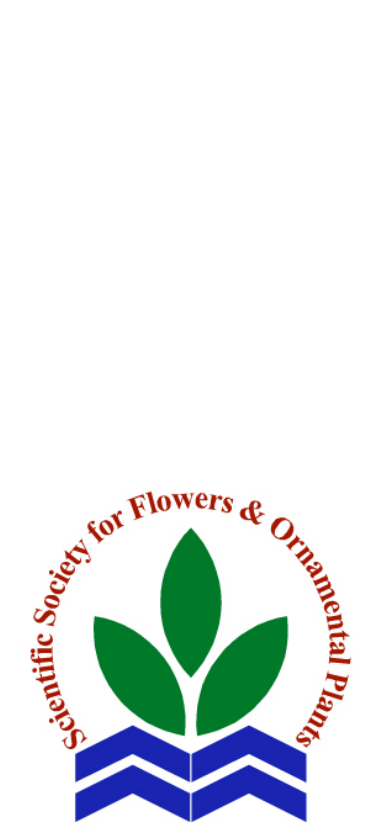

Scientific J. Flowers \& Ornamental Plants, 3(4):215-232 (2016).

Received:

$1 / 9 / 2016$

Revised by:

Prof. Dr. E.S. Nofal, Kafr El-Sheikh Univ.

Prof. Dr. A.Z. Sarhan, Cairo Univ.
ABSTRACT: Nowadays, the most of investigations are directed towards the using of natural materials for reducing pollution environment. So, this study was consummated at Post-Harvest Lab. of Floriculture Dept., Hort. Res. Inst., Giza, Egypt during 2012 and 2013 seasons to study the effect of some holding solutions, viz. distilled water (control), calcium chloride ( $1 \& 3 \mathrm{~g} / \mathrm{l})$, calcium claw ( $1 \& 3 \mathrm{~g} / \mathrm{l}$ ), egg shell ( $0.5 \& 1.5 \mathrm{~g} / \mathrm{l})$, oysters ( $0.5 \& 1.5 \mathrm{~g} / \mathrm{l}$ ), humic acid (50 \& 150 $\mathrm{g} / \mathrm{l})$ and three doses of gamma irradiation (100, $200 \& 400 \mathrm{~Gy})$ on longevity of flower heads (vase life), water uptake, water loss, relative fresh weight, dry weight and flower diameter of Gerbera jamesonii, cv. Rosalin \& Express cut flowers. The obtained results cleared that application of $\mathrm{CaCl}_{2}$ ( 1 or $3 \mathrm{~g} / \mathrm{l}$ ) with HQ $0.2 \mathrm{~g} / \mathrm{l}$ and $20 \mathrm{~g} / \mathrm{l}$ sucrose resulted in a great extension in vase life and water uptake more than other treatments. Rosalin cultivar recorded significant increase in longevity, water uptake comparing to gerbera cut flowers of $\mathrm{cv}$. Express during the two seasons. Gamma ray (200 Gy) recorded the least rate of water loss in cv. Rosalin in the two seasons. Superiority was for the treatment with oysters $(1.5 \mathrm{~g} / \mathrm{l})$ in improvement of relative fresh weight in both seasons followed by gamma ray (200 Gy), also gamma irradiation at the doses $200 \mathrm{~Gy}$ plus $\mathrm{CaCl}_{2}$ at $1 \mathrm{~g} / \mathrm{l}+$ sucrose at $20 \mathrm{~g} / \mathrm{l}$ significantly increased dry weight of the two cultivars Rosalin and Express followed by claw $(1 \mathrm{~g} / \mathrm{l})$. All treatments gave higher record in comparison with control for diameter. The present research indicated that the application of $\mathrm{CaCl}_{2}(1 \mathrm{~g} / \mathrm{l})$ with HQ $0.2 \mathrm{~g} / \mathrm{l}$ and 20 $\mathrm{g} / \mathrm{l}$ sucrose after exposure of cut flowers to irradiation with gamma at (200 Gy) maximizes flower diameter. Results showed that, treating with $\mathrm{CaCl}_{2}$ at $3 \mathrm{~g} / \mathrm{l}+\mathrm{HQ}$ at $0.2 \mathrm{~g} / \mathrm{l}$ and suc. at $20 \mathrm{~g} / \mathrm{l}$, or gamma rays at $200 \mathrm{~Gy}+\mathrm{CaCl}_{2}$ at $1 \mathrm{~g} / \mathrm{l}+\mathrm{HQ}$ at $2 \mathrm{~g} / \mathrm{l}+$ Sucrose at $20 \mathrm{~g} / \mathrm{l}$ registered the highest increments in chlorophyll a in the stems and soluble sugars in the stems and petals. It is concluded that the use of the holding solution containing calcium chloride at $3 \mathrm{~g} / \mathrm{l}$ plus hydroxyqenolin plus sucrose alone or after irradiation with gamma at 200 Gy gave the maximum vase life.

Key words: Vase life Gerbera jamesonii, cv. Rosalin \& Express, calcium chloride, calcium claw, egg shell, oysters, humic acid, gamma irradiation, water uptake, water loss.

\section{INTRODUCTION}

Gerbera is a genus of ornamental plants from the family Asteraceae. It has approximately 30 species in the wild, extending to South American, Africa and tropical Asia. Besides floral arrangements, gerbera is widely used in bouquets. Gerbera jamesonii is one of the ten most popular commercial cut flowers in the world and according to the global trends in floriculture, 
it occupies the fourth place among cut flowers (Choudhary and Prasad, 2000).

Gerberas are valued for their brightly coloured daisy like flowers. Flowers are available in a wide range of colors, including yellow, orange, pink, crimson, red, purple and white. Gabera jamesonii is native to South Africa (Transvaal and Natal Provinces) and Swaziland (Emongor, 2004). Dineshbabu et al. (2002) reported that holding solutions containing 8-HQS + sucrose extended the vase life of Dendrobium flowers and improved flower quality, as suggested by improved water consumption, fresh weight and flower freshness. This treatment also reduced the respiration rate and physiological loss in weight.

Chen et al. (2004) pointed out that cut flowers of Gerbera hybrida were steeped in $\mathrm{CaCl}_{2}$ solution and their flowering period had been prolonged.

Application of $\mathrm{CaCl}_{2}$ extended the vase life of flowers and reduced flowers bending of gerbera (Gerasopoulos and Chebli, 1999).

Bulent and Senay (2012) found that Ca treatment with NA and MA significantly reduced postharvest quality losses of gerbera (Gerbera jamesonii cv. Rosalin). Chutichudet and Chutichudet (2012) reported that, applications of $\mathrm{CaCl}_{2}$ at 0.4 and $0.8 \%$ were found to be not the appropriate substances for maintaining the quality and prolonging the vase life of the patumma's flower.

Samaneh et al. (2015) found that 8-HQS increased vase life, dry weight, flower diameter, mean absorbed preservative solution and quality score. Also, decreased the stem curvature. Sucrose decreased vase life, flower diameter and quality score, whereas increased dry weight of cut Gerbera flower.

The egg shell consists $95 \%$ of the weight of calcium carbonate and $3.5 \%$ of the remaining organic material is a mixture according to Daengprok et al. (2003).
Oysters are an excellent source of zinc, iron, calcium, and selenium, as well as vitamin $\mathrm{A}$ and vitamin $\mathrm{B}_{12}$. Oysters are low in food energy; one dozen raw oysters contains 110 kilocalories (460 kJ). Mollusks (2011).

Dietary supplements may contain calcium carbonate from oyster shells, though no evidence shows this offers any benefits beyond what calcium may offer. The postharvested vegetables can be irradiated with gamma rays or electron beam to attend the phytosanitary requirements. Some previous researches indicate the effectiveness of radiation for disinfestations of flowers (Hayashi et al., 1998; Kikuchi, 2000; Tanabe and Dohino, 1995). Placing chrysanthemum stems in $2 \%$ sucrose before and during irradiation did not influence the vase-life, but placing chrysanthemum stems in $2 \%$ sucrose following irradiation prolonged the vase-life (Hayashi and Dohino, 1995; Hayashi and Todoriki, 1996). These results indicate that sugars influence post-irradiation metabolism responsible for radiation-induced deterioration of chrysanthemum cut flowers. Dohino and Hayashi (1995) cleared that irradiation reduced ethylene production by cut flower.

Uthairatanakij et al. (2005) found that after harvesting, inflorescences were held in distilled water or holding solution containing $4 \%$ glucose $+220 \mathrm{mg} / \mathrm{l} \mathrm{HQS}+30 \mathrm{mg} / \mathrm{l}$ $\mathrm{AgNO}_{3}$ and irradiated with gamma ray at 2.5 kGy. $\mathrm{CaCl}_{2}$ spraying reduced flower dropping compared to control treatment. The beneficial vase solutions on irradiated flowers were to increase bud opening and reduce respiration rate, ethylene production and prolonged vase life up to 3 days at $25^{\circ} \mathrm{C}$.

Miscellaneous evidence has demonstrated that humic acid plays an important regulatory role in multiple plant physiological processes as a plant growth regulator and delays the process of senescence in flowers (Nardi et al., 2002). A pulse treatment of the flower with $1000 \mathrm{ppm}$ humic acid was found as the most suitable treatment in preventing the flower from leaf 
yellowing (Keshavarzi and Chamani, 2011). Vase life for up to 10 days and petal protein contents (32.76\%) in Chrysanthemum (Dendranthema grandiflorum) flower was improved with respect to control when humic acid was applied with another compound Samiee et al. (2013). Kikuchi et al. (1995) irradiated Yellow minichrysanthemums in a Cobalt-60 gamma cell at the dose of $900 \mathrm{~Gy}(467 \mathrm{~Gy} / \mathrm{h})$ one day after harvest. Samples of 50 flowers, partially opened buds were used to estimate the flower viability. Aluminum sulfate and 8-hydroxyquinoline sulfate were used as two preservative solutions aiming to protect the cut flowers. Results indicated that the stem immersion in the preservative solutions after the irradiation treatment was an efficient procedure, stimulating the flowers development and maintaining the vase-life almost as long as the controls.

It would be possible to use preservative solutions to minimize the damaging effects of the ionizing radiation on chrysanthemum cut flowers, maintaining at the same time the disinfestations action of radiation process. Radiation could be an effective treatment against insects that will prevent their reproduction in the host country. The radiation treatment could be also an alternative to substitute chemical fumigation with methyl bromide, which destroys the ozone layer of the earth.

The cut flower is an alive organism and in some cases it continues developing during its vase-life. It can be composed of one or many flowers, and may include leaves and stem, or only flower and stem. Some are harvested as bud and others after the blossom. The vase-life varies with the species or cultivar. Handling and environmental conditions during the vaselife can also affect flower vigor. Infestation by pests can drastically damage the final product, so that it can be considered commercially unsuitable. Flowers do not differ from other biological organisms in that they can be damaged by radiation, depending on the dose. Some authors have irradiated cut flowers with gamma-radiation [Haasbroek, et al., 1973; Hayashi, 1996], while others have used electron beams [Tanabe and Dohino, 1993 and 1995] for dis infestation. To avoid the deleterious effects of radiation, cut flowers can be supplied with preservative or holding solutions containing sugar [Haasbroek et al., 1973; Kikuchi, 1995; Hayashi, 1996]. Unfortunately, this procedure also must be adopted by the florist and the final consumer to assure continued flower quality during the vase-life.

Hamidah et al. (2006) found that, tolerant dose of cut flowers is the highest dose that can be applied to the plant without any visible injuries in terms of quality and appearance. The tolerant dose for roses is $100 \mathrm{~Gy}$, for carnation $200 \mathrm{~Gy}$, for orchids 100-300 Gy depending on the variety tested. The cut chrysanthemum can tolerate 200-400 Gy depending on the variety tested. However, chrysanthemum in $4 \%$ sucrose solution can tolerate doses of 750 Gy. Based on the obtained results, the quarantine sterilizing dose for tetranychuspiercie is 350 Gy and it is only suitable for chrysanthemums. Similar observations were also gained by Mencarelli et al. (1995); Amariutei et al. (1995); Nagaraja et al. (2000) and Emongor (2004) on gerbera, as well as Celikel and Karaaly (1995) on carnation, Reyes-Arribas et al. (2000) on Chrysanthemum cvs. Tara and Boaldi and Singh and Tiwari (2002) on rose cv. Dorris.

This trial aims to examine the effect of some holding solutions which contained either of calcium ,egg shell, oysters, humic acid and gamma irradiation amended with sucrose on quality, longevity, water relations and stem and petal chemical composition of cut gerbera (cv. Rosalin and cv. Express) flower heads .

\section{MATERIALS AND METHODS}

A study was conducted at Post-Harvest Lab. of Floriculture Dept., Hort. Res. Inst., Giza, Egypt, during 2012 and 2013 seasons in order to determine the best treatment suitable for improving the flowers keeping 
quality and detecting some morphological and physiological changes occurring during the vase life period of the cut Gerbera flowers.

Flower heads of Gerbera jamesonii, cvs. Rosalin \& Express were freshly obtained from Floramix Farm (El-Mansouria, Giza) grown under standard cultural practices in a commercial greenhouse. The flower heads were picked in the early morning at the export stage. Immediately following harvest ( $1 \mathrm{~h})$ flowers were transported to the laboratory. The end of stems were recut and placed in graduated cylinders (3flower heads/cylinder) containing one of the following holding solutions:

1- Distilled water (referred to as control).

2- $\mathrm{CaCl}_{2}(1 \mathrm{~g} / \mathrm{l})+\mathrm{HQ}(0.2 \mathrm{~g} / \mathrm{l})+$ sucrose $(20$ $\mathrm{g} / \mathrm{l})$ solution.

3- $\mathrm{CaCl}_{2}(3 \mathrm{~g} / \mathrm{l})+\mathrm{HQ}(0.2 \mathrm{~g} / \mathrm{l})+$ sucrose $(20$ g/l) solution.

4- Egg shell (0.50 g/l) + HQ (0.2 g/l) + sucrose $(20 \mathrm{~g} / \mathrm{l})$ solution.

5- Egg shell (1.50 g/l) + HQ (0.2 g/l) + sucrose (20 g/l) solution.

6- Calcium claw (1 g/l) + HQ (0.2 g/l) + sucrose (20 g/l) solution.

7- Calcium claw (3 g/l) + HQ (0.2 g/l) + sucrose (20 g/l) solution.

8- Oysters $(0.50 \mathrm{~g} / \mathrm{l})+\mathrm{HQ}(0.2 \mathrm{~g} / \mathrm{l})+$ sucrose $(20 \mathrm{~g} / \mathrm{l})$ solution.

9- Oysters (1.50 g/l) + HQ (0.2 g/l) + sucrose $(20 \mathrm{~g} / \mathrm{l})$ solution.

10- Humic acid (actosol®) $(50 \mathrm{~g} / \mathrm{l})+\mathrm{CaCl}_{2}$ $(1 \mathrm{~g} / \mathrm{l})+$ sucrose $(20 \mathrm{~g} / \mathrm{l})$ solution .

11- Humic acid (actosol $\AA)(150 \mathrm{~g} / \mathrm{l})+\mathrm{CaCl}_{2}$ $(1 \mathrm{~g} / \mathrm{l})+$ sucrose $(20 \mathrm{~g} / \mathrm{l})$ solution.

12- Gamma rays at $100 \mathrm{~Gy}+\mathrm{CaCl}_{2}(1 \mathrm{~g} / \mathrm{l})+$ HQ $(0.2 \mathrm{~g} / \mathrm{l})+$ sucrose $(20 \mathrm{~g} / \mathrm{l})$.

13- Gamma rays at $200 \mathrm{~Gy}+\mathrm{CaCl}_{2}(1 \mathrm{~g} / \mathrm{l})+$ HQ $(0.2 \mathrm{~g} / \mathrm{l})+$ sucrose $(20 \mathrm{~g} / \mathrm{l})$.

14- Gamma rays at $400 \mathrm{~Gy}+\mathrm{CaCl}_{2}(1 \mathrm{~g} / \mathrm{l})+$ HQ $(0.2 \mathrm{~g} / \mathrm{l})+$ sucrose $(20 \mathrm{~g} / \mathrm{l})$.
The chemical composition of egg shell was $91.1 \%$ ash, $7.56 \%$ protein, $0.24 \%$ lipid, $36.4 \%$ calcium, $0.002 \%$ iron, $0.097 \%$ potassium, $\quad 0.398 \%$ magnesium, $0.152 \%$ sodium, $\quad 0.091 \%$ sulfur, and $0.116 \%$ phosphorus (Walton et al., 1973).

Oysters are an excellent source of zinc, iron, calcium, and selenium, as well as vitamin $A$ and vitamin $B_{12}$. Oysters are low in food energy; one dozen raw oysters contain 110 kilocalories (460 kJ) (Mollusks, 2011). Humic acid (commercial liquid organic fertilizer (micronutrients actosol ${ }^{\circledR}$ ) containing $2.9 \%$ humic acid plus $0.5 \%$ from each of $\mathrm{Fe}, \mathrm{Zn}, \mathrm{Mn}$ and $\mathrm{Cu}$ ).

Stalks were irradiated at the Middle Estern Regional Radioistope Center for the Arab Countries, Dokki, Giza using gamma cell (Co 60) at 0, 100, 200, 400 Gy for 6 min and 14 sec.

The flower heads were held under a $24 \mathrm{~h}$ photoperiod (fluorescent light of 1000 lux) at $20 \pm 1{ }^{\circ} \mathrm{C}$ and approx $85 \%$ relative humidity. During the vase life period, some data were recorded every three days as follows: longevity (vase life) (days), water uptake (g/flower), water loss(g/flower), flower diameters $(\mathrm{cm})$, relative fresh weight $(\%)$ and dry weight (\%).

Chlorophhyll a, b and carotenoids (mg/g f.w.) content were determined in fresh stem samples according to the method of Saric et al. (1967), while in fresh petal samples, carotenoids content was only evaluated. In fresh stems and petal samples, however, total soluble sugars (g/100 g) were measured as described by Dubois et al. (1956).

The design used was a completely randomized design in a factorial experiment as described by Snedecor and Cochran, (1972) at 5\% probability level. Data obtained were statistically analyzed using MSTAT Computer Program (1985). Means were compared by Duncan's multiple range test (1955), which was used to verify the significance level among means of various treatments. 


\section{RESULTS AND DISCUSSION}

\section{Effect of holding solutions on:}

\section{Vase life of cut flower heads:}

Data presented in Table (1) show that flower stems kept in water containing $\mathrm{CaCl}_{2}$, Ca claw, oysters had significantly increased vase life relative to the control for all concentrations in both cultivar Rosalin \& Express in the two seasons and application of $\mathrm{CaCl}_{2}(1$ or $3 \mathrm{~g} / \mathrm{l}$ ) with HQ at $0.2 \mathrm{~g} / \mathrm{l}$ and $20 \mathrm{~g} / \mathrm{l}$ sucrose resulted in a greater extension in vase life than other treatments, these results are in accordance with those reported (Cortes et al., 2011) that treatment with $\mathrm{CaCl}_{2}+$ suc. + HQS also had the greatest vase life in Rosa hybrida cv. Grand Gala. It is concluded that the combined effect of $\mathrm{Ca}$ as a flow resistance reducer and HQS as a germicidal agent contributed to improve vase life. (Ibrahim et al., 2011) found that, The chemical solutions of 8-HQS at 100 or 200 ppm or $\mathrm{CaCl}_{2}$ at 1000 or 2000 ppm each supplemented with 4\% sucrose increased longevity of gerbera cut flowers, Dineshbabu et al. (2002) reported that holding solutions containing 8-HQS + sucrose extended the vase life of Dendrobium flowers.

Gamma (200 Gy) + $\mathrm{CaCl}_{2}+\mathrm{HQ}+$ suc. was the best treatment between radiation the two cultivars in all seasons and gave a significant finding, this agreed with the results found by (Hayashi and Todoriki, 1996) that holding chrysanthemum cut flowers in a sucrose solution before and during irradiation did not influence the vase life, but holding the cut flowers in a sucrose solution following irradiation prolonged the vase life. Uthairatanakij et al. (2005) on cut Dendrobium flowers demonstrated that, the beneficial effects on vase solutions in irradiated flowers were to increase bud opening and reduced respiration rate, ethylene production and prolonged vase life up. However, the shortest vase life in cv. Rosalin was given by humic acid in the two doses used, these results are in harmony with those of the shortest vase life were related to humic acid in cut roses (Mojgan et al.,
2013). Vase life of gerbera cut flowers differed among the different treatments. Maximum vase life in Rosalin cultivar (15 d) was noticed due to $\mathrm{CaCl}_{2}$ (3 g/ l)+ HQ (0.2 $\mathrm{g} / \mathrm{l})+$ sucrose $(20 \mathrm{~g} / \mathrm{l})$ while the maximum vase life in Express cultivar (14 d) was noticed in two treatments $\mathrm{CaCl}_{2}(3 \mathrm{~g} / \mathrm{l})+\mathrm{HQ}$ $(0.2 \mathrm{~g} / \mathrm{l})+$ sucrose $(20 \mathrm{~g} / \mathrm{l})$ solution and egg shell $(1.50 \mathrm{~g} / \mathrm{l})+\mathrm{HQ}(0.2 \mathrm{~g} / \mathrm{l})+$ sucrose (20 g/l) solution. In cv. Express all treatments gave maximum value over than control treatment giving the minimum value in both seasons.

Regarding the effect of gerbera varieties Rosalin and Express on longevity of flower heads (days), Rosalin cultivar (Table, 1) recorded highly significant increase in longevity comparing to gerbera cut flowers cv. Express during the two seasons, the results were in harmony with the findings of Nazari et al. (2011) who indicated that many cultivars of gerbera had high vase life, while many cultivar had low vase life and some of them had moderate vase life. On the other hand, other cultivars had minimum vase life. Emongor (2004) demonstrated that genetic factor has a critical role in vase life of gerbera.

\section{Water uptake:}

Water uptake as shown in Table (2) recorded maximum value in $\mathrm{CaCl}_{2}(3 \mathrm{~g} / \mathrm{l})+$ $\mathrm{HQ}+$ suc. (75.24 g/l) in cv. Rosalin, and $\mathrm{CaCl}_{2}(1 \mathrm{~g} / \mathrm{l})+\mathrm{HQ}+$ suc. $(52.13 \mathrm{~g} / \mathrm{l})$ in $\mathrm{cv}$. Express that was cleared in the second season as treatment with $\mathrm{CaCl}_{2}$ achieved significant increasing in water uptake in both cultivars (84.20 in cv. Rosalin, 56.82 in cv. Express). On comparison of both cultivars for water uptake cv. Rosalin was found superior over cv. Express in the two seasons. In this concern, Dineshbabu et al. (2002) reported that holding solutions containing 8-HQS + sucrose improved flower of Dendrobium flowers quality, as suggested by improved water consumption. Similarly Cortes et al. (2011) on cut flowers of rose mentioned that the $\mathrm{CaCl}_{2}+$ suc. + HQS treatment induced a greater water flow and the most color infiltration through the 
Table 1. Effect of holding solution treatments on vase life of Gerbera jamesonii, cv. Rosalin and Express flower heads (days) during the vase life period of 2012 and 2013 seasons.

\begin{tabular}{|c|c|c|c|}
\hline Treatments & Rosalin & Express & Mean \\
\hline \multicolumn{4}{|c|}{ First season: 2012} \\
\hline D.W. (Control) & $10.00 \mathrm{ef}$ & $7.00 \mathrm{~h}$ & $8.50 \mathrm{e}$ \\
\hline $\mathrm{CaCl}_{2} 1 \mathrm{~g} / \mathrm{l}+\mathrm{HQ} 0.2 \mathrm{~g} / \mathrm{l}+$ suc. $20 \mathrm{~g} / \mathrm{l}$ & $14.00 \mathrm{ab}$ & 13.00bc & $13.50 \mathrm{a}-\mathrm{c}$ \\
\hline $\mathrm{CaCl}_{2} 3 \mathrm{~g} / \mathrm{l}+\mathrm{HQ} 0.2 \mathrm{~g} / \mathrm{l}+$ suc. $20 \mathrm{~g} / \mathrm{l}$ & $15.00 \mathrm{a}$ & $14.00 \mathrm{ab}$ & $14.50 \mathrm{a}$ \\
\hline Egg shell 0.5 g/l + HQ 0.2 g/l + suc. 20 g/l & 14.00ab & 14.00ab & $14.00 \mathrm{ab}$ \\
\hline Egg shell 1.5 g/l + HQ 0.2 g/l + suc. 20 g/l & 11.50c-e & 14.00ab & $12.75 b-c$ \\
\hline Ca claw 1 g/l + HQ 0.2 g/l + suc. 20 g/l & $14.00 \mathrm{ab}$ & $9.00 \mathrm{fg}$ & $11.50 \mathrm{~d}$ \\
\hline Ca claw 3 g/l + HQ 0.2 g/l + suc. 20 g/l & $14.50 \mathrm{ab}$ & 11.50c-e & $13.00 \mathrm{bc}$ \\
\hline Oysters 0.5 g/l + HQ 0.2 g/l + suc. 20 g/l & $12.00 \mathrm{~cd}$ & 11.00de & $11.50 \mathrm{~d}$ \\
\hline Oysters 1.5 g/l + HQ 0.2 g/l + suc. 20 g/l & $14.17 \mathrm{ab}$ & $10.67 d-f$ & $12.42 \mathrm{~cd}$ \\
\hline Humic acid 50 g/l + $\mathrm{CaCl}_{2} 1 \mathrm{~g} / \mathrm{l}+$ suc. $20 \mathrm{~g} / \mathrm{l}$ & $9.00 \mathrm{fg}$ & $8.00 \mathrm{gh}$ & $8.5 e$ \\
\hline Humic acid 150 g/l + $\mathrm{CaCl}_{2} 1 \mathrm{~g} / \mathrm{l}+$ suc. $20 \mathrm{~g} / \mathrm{l}$ & $9.00 \mathrm{fg}$ & $9.00 \mathrm{fg}$ & $9.00 \mathrm{e}$ \\
\hline Gamma $100 \mathrm{~Gy}+\mathrm{CaCl}_{2} 1 \mathrm{~g} / \mathrm{l}+\mathrm{HQ} 0.2 \mathrm{~g} / \mathrm{l}+$ suc. $20 \mathrm{~g} / \mathrm{l}$ & $9.00 \mathrm{fg}$ & 10.50d-f & $9.75 \mathrm{e}$ \\
\hline Gamma $200 \mathrm{~Gy}+\mathrm{CaCl}_{2} 1 \mathrm{~g} / \mathrm{l}+\mathrm{HQ} 0.2 \mathrm{~g} / \mathrm{l}+$ suc. $20 \mathrm{~g} / \mathrm{l}$ & $14.00 \mathrm{ab}$ & 14.00ab & $14.00 \mathrm{ab}$ \\
\hline Gamma $400 \mathrm{~Gy}+\mathrm{CaCl}_{2} 1 \mathrm{~g} / \mathrm{l}+\mathrm{HQ} 0.2 \mathrm{~g} / \mathrm{l}+$ suc. $20 \mathrm{~g} / \mathrm{l}$ & $10.00 \mathrm{eg}$ & $9.00 \mathrm{fg}$ & $9.50 \mathrm{e}$ \\
\hline Mean & $12.15 \mathrm{a}$ & $11.05 \mathrm{~b}$ & \\
\hline
\end{tabular}

Second season: 2013

D.W. (Control)

$\mathrm{CaCl}_{2} 1 \mathrm{~g} / \mathrm{l}+\mathrm{HQ} 0.2 \mathrm{~g} / \mathrm{l}+$ suc. $20 \mathrm{~g} / \mathrm{l}$

$\mathrm{CaCl}_{2} 3 \mathrm{~g} / \mathrm{l}+\mathrm{HQ} 0.2 \mathrm{~g} / \mathrm{l}+$ suc. $20 \mathrm{~g} / \mathrm{l}$

Egg shell 0.5 g/l + HQ 0.2 g/l + suc. 20 g/l

Egg shell 1.5 g/l + HQ 0.2 g/l + suc. 20 g/l

Ca claw 1 g/l + HQ 0.2 g/l + suc. 20 g/l

Ca claw 3 g/l + HQ 0.2 g/l + suc. 20 g/l

Oysters 0.5 g/l + HQ 0.2 g/l + suc. 20 g/l

Oysters 1.5 g/l + HQ 0.2 g/l + suc. 20 g/l

Humic acid 50 g/l + $\mathrm{CaCl}_{2} 1 \mathrm{~g} / \mathrm{l}+$ suc. $20 \mathrm{~g} / \mathrm{l}$

Humic acid 150 g/l $+\mathrm{CaCl}_{2} 1 \mathrm{~g} / \mathrm{l}+$ suc. $20 \mathrm{~g} / \mathrm{l}$

Gamma $100 \mathrm{~Gy}+\mathrm{CaCl}_{2} 1 \mathrm{~g} / \mathrm{l}+\mathrm{HQ} 0.2 \mathrm{~g} / \mathrm{l}+$ suc. $20 \mathrm{~g} / \mathrm{l}$

Gamma $200 \mathrm{~Gy}+\mathrm{CaCl}_{2} 1 \mathrm{~g} / \mathrm{l}+\mathrm{HQ} 0.2 \mathrm{~g} / \mathrm{l}+$ suc. $20 \mathrm{~g} / \mathrm{l}$

Gamma $400 \mathrm{~Gy}+\mathrm{CaCl}_{2} 1$ g/l + HQ 0.2 g/l + suc. 20 g/l

Mean

Means having the same letter are not significantly differed at .05 level of probability.

\begin{tabular}{ccc}
$10.00 \mathrm{~cd}$ & $7.00 \mathrm{f}$ & $8.50 \mathrm{e}$ \\
$14.00 \mathrm{ab}$ & $13.00 \mathrm{~b}$ & $13.50 \mathrm{a}$ \\
$14.00 \mathrm{ab}$ & $14.00 \mathrm{ab}$ & $14.00 \mathrm{a}$ \\
$14 . \mathrm{ab}$ & $14.00 \mathrm{ab}$ & $14.00 \mathrm{a}$ \\
$13.00 \mathrm{~b}$ & $14.00 \mathrm{ab}$ & $13.50 \mathrm{a}$ \\
$14.00 \mathrm{ab}$ & $9.00 \mathrm{de}$ & $11.50 \mathrm{~b}$ \\
$15.00 \mathrm{a}$ & $8.00 \mathrm{ef}$ & $11.50 \mathrm{~b}$ \\
$14.00 \mathrm{ab}$ & $8.00 \mathrm{ef}$ & $11.00 \mathrm{bc}$ \\
$13.00 \mathrm{~b}$ & $13.00 \mathrm{~b}$ & $13.00 \mathrm{a}$ \\
$9.00 \mathrm{de}$ & $8.00 \mathrm{ef}$ & $8.50 \mathrm{e}$ \\
$9.00 \mathrm{de}$ & $9.00 \mathrm{de}$ & $9.00 \mathrm{de}$ \\
$9.00 \mathrm{de}$ & $9.00 \mathrm{de}$ & $9.00 \mathrm{de}$ \\
$14.00 \mathrm{ab}$ & $14.00 \mathrm{ab}$ & $14.00 \mathrm{a}$ \\
$11.00 \mathrm{c}$ & $9.00 \mathrm{de}$ & $10.00 \mathrm{~cd}$ \\
12.36 & $10.64 \mathrm{~b}$ & \\
\hline
\end{tabular}


Table 2. Effect of holding solution treatments on water uptake of Gerbera jamesonii, cv. Rosalin and Express flower heads (g/flower head) during the vase life period of 2012 and 2013 seasons.

\begin{tabular}{|c|c|c|c|}
\hline Treatments & Rosalin & Express & Mean \\
\hline \multicolumn{4}{|c|}{ First season: 2012} \\
\hline D.W. (Control) & 65.43ab & $50.95 c-h$ & 58.19ab \\
\hline $\mathrm{CaCl}_{2} 1 \mathrm{~g} / \mathrm{l}+\mathrm{HQ} 0.2 \mathrm{~g} / \mathrm{l}+$ suc. $20 \mathrm{~g} / \mathrm{l}$ & 59.37bc & 52.13c-g & $55.75 a-c$ \\
\hline $\mathrm{CaCl}_{2} 3 \mathrm{~g} / \mathrm{l}+\mathrm{HQ} 0.2 \mathrm{~g} / \mathrm{l}+$ suc. $20 \mathrm{~g} / \mathrm{l}$ & $75.24 a$ & 48.15d-h & $61.70 \mathrm{a}$ \\
\hline Egg shell 0.5 g/l + HQ 0.2 g/l + suc. 20 g/l & 57.36b-f & $51.11 \mathrm{c}-\mathrm{h}$ & $54.24 a-d$ \\
\hline Egg shell 1.5 g/l + HQ 0.2 g/l + suc. 20 g/l & $53.77 \mathrm{c}-\mathrm{g}$ & $48.26 \mathrm{~d}-\mathrm{h}$ & $51.02 b-e$ \\
\hline Ca claw 1 g/l + HQ 0.2 g/l + suc. 20 g/l & $46.20 \mathrm{~g}-\mathrm{k}$ & 43.93g-l & $45.07 \mathrm{e}-\mathrm{h}$ \\
\hline Ca claw 3 g/l + HQ 0.2 g/l + suc. 20 g/l & $58.26 b-d$ & 49.61c-h & $53.94 b-c$ \\
\hline Oysters 0.5 g/l + HQ 0.2 g/l + suc. 20 g/l & 57.86b-e & 37.38i-n & $47.62 d-g$ \\
\hline Oysters 1.5 g/l + HQ 0.2 g/l + suc. 20 g/l & $57.10 \mathrm{~b}-\mathrm{f}$ & 40.80h-m & $48.95 c-f$ \\
\hline Humic acid $50 \mathrm{~g} / \mathrm{l}+\mathrm{CaCl}_{2} 1 \mathrm{~g} / \mathrm{l}+$ suc. $20 \mathrm{~g} / \mathrm{l}$ & $47.30 \mathrm{e}-\mathrm{i}$ & 34.991- & 41.15gh \\
\hline Humic acid 150 g/l + $\mathrm{CaCl}_{2} 1 \mathrm{~g} / \mathrm{l}+$ suc. $20 \mathrm{~g} / \mathrm{l}$ & 49.99c-h & $27.74 n$ & $38.87 \mathrm{~h}$ \\
\hline Gamma $100 \mathrm{~Gy}+\mathrm{CaCl}_{2} 1 \mathrm{~g} / \mathrm{l}+\mathrm{HQ} 0.2 \mathrm{~g} / \mathrm{l}+$ suc. $20 \mathrm{~g} / \mathrm{l}$ & 46.26g-j & $32.2 \mathrm{mn}$ & 39.23h \\
\hline Gamma $200 \mathrm{~Gy}+\mathrm{CaCl}_{2} 1 \mathrm{~g} / \mathrm{l}+\mathrm{HQ} 0.2 \mathrm{~g} / \mathrm{l}+$ suc. $20 \mathrm{~g} / \mathrm{l}$ & 40.58h-m & $35.62 k-n$ & 38.10h \\
\hline Gamma $400 \mathrm{~Gy}+\mathrm{CaCl}_{2} 1 \mathrm{~g} / \mathrm{l}+\mathrm{HQ} 0.2 \mathrm{~g} / \mathrm{l}+$ suc. $20 \mathrm{~g} / \mathrm{l}$ & 47.09f-i & $36.30 \mathrm{j}-\mathrm{n}$ & 41.70f-h \\
\hline Mean & $54.42 \mathrm{a}$ & $42.08 \mathrm{~b}$ & \\
\hline \multicolumn{4}{|c|}{ Second season: 2013} \\
\hline D.W. (Control) & $63.07 \mathrm{~d}$ & $52.17 \mathrm{gh}$ & 57.62c \\
\hline $\mathrm{CaCl}_{2} 1 \mathrm{~g} / \mathrm{l}+\mathrm{HQ} 0.2 \mathrm{~g} / \mathrm{l}+$ suc. $20 \mathrm{~g} / \mathrm{l}$ & 58.06e & $53.64 g$ & $55.85 d$ \\
\hline $\mathrm{CaCl}_{2} 3 \mathrm{~g} / \mathrm{l}+\mathrm{HQ} 0.2 \mathrm{~g} / \mathrm{l}+$ suc. $20 \mathrm{~g} / \mathrm{l}$ & $84.20 \mathrm{a}$ & 56.82ef & $70.51 \mathrm{a}$ \\
\hline Egg shell 0.5 g/l + HQ 0.2 g/l + suc. 20 g/l & $55.28 f$ & $57.04 \mathrm{e}$ & $56.16 \mathrm{~d}$ \\
\hline Egg shell 1.5 g/l + HQ 0.2 g/l + suc. 20 g/l & 52.22gh & $50.02 \mathrm{ij}$ & $51.12 \mathrm{f}$ \\
\hline Ca claw 1 g/l + HQ 0.2 g/l + suc. 20 g/l & 43.031 & $43.21 \mathrm{kl}$ & 43.12h \\
\hline Ca claw 3 g/l + HQ 0.2 g/l + suc. 20 g/l & $58.40 \mathrm{e}$ & 42.14lm & $50.27 f$ \\
\hline Oysters 0.5 g/l + HQ 0.2 g/l + suc. 20 g/l & 67.32c & $40.82 \mathrm{~m}$ & 54.07e \\
\hline Oysters 1.5 g/l + HQ 0.2 g/l + suc. 20 g/l & $72.16 b$ & 50.84hi & $61.50 \mathrm{~b}$ \\
\hline Humic acid 50 g/l + $\mathrm{CaCl}_{2} 1 \mathrm{~g} / \mathrm{l}+$ suc. $20 \mathrm{~g} / \mathrm{l}$ & $44.82 \mathrm{k}$ & 31.010 & $37.92 \mathrm{j}$ \\
\hline Humic acid 150 g/l + $\mathrm{CaCl}_{2} 1 \mathrm{~g} / \mathrm{l}+$ suc. $20 \mathrm{~g} / \mathrm{l}$ & $55.30 f$ & $22.19 q$ & 38.75ij \\
\hline Gamma $100 \mathrm{~Gy}+\mathrm{CaCl}_{2} 1 \mathrm{~g} / \mathrm{l}+\mathrm{HQ} 0.2 \mathrm{~g} / \mathrm{l}+$ suc. $20 \mathrm{~g} / \mathrm{l}$ & $48.60 \mathrm{j}$ & $42.00 \mathrm{~lm}$ & $45.30 \mathrm{~g}$ \\
\hline Gamma $200 \mathrm{~Gy}+\mathrm{CaCl}_{2} 1 \mathrm{~g} / \mathrm{l}+\mathrm{HQ} 0.2 \mathrm{~g} / \mathrm{l}+$ suc. $20 \mathrm{~g} / \mathrm{l}$ & $37.79 n$ & 29.660 & $33.73 \mathrm{k}$ \\
\hline Gamma $400 \mathrm{~Gy}+\mathrm{CaCl}_{2} 1$ g/l + HQ 0.2 g/l + suc. 20 g/l & $52.95 g$ & 25.33p & $39.14 \mathrm{i}$ \\
\hline Mean & 56.66a & 42.64 & \\
\hline
\end{tabular}

Means having the same letter are not significantly differed at .05 level of probability. 
vascular tissue compared to the sucrose treatment alone. Van Doorn and Witte (1994) pointed out that in gerbera cut flowers, It can be explained that application of these chemical preservatives 8-HQS, calcium chloride probably reduced microbial contamination in flower stems or vase water and consequently improved water conductance by preventing bacterial growth and reducing occlusions, also Ichimura et al. (2005) reported that the treatment with HQS improved water consumption and this was likely due to its acidifying effect that inhibits vascular occlusion caused by microorganisms .however, the lowest was recorded by gamma (200 Gy) $+\mathrm{CaCl}_{2}(1 \mathrm{~g} / \mathrm{l})$ $+\mathrm{HQ}+$ suc. in cv. Rosalin in the two seasons, and treatment with HA (150 g/l + $\mathrm{CaCl}_{2}+$ suc.) gave the lowest value of absorbent of solution in CV. Express in both seasons Mojgan et al. (2013) pointed out that a solution of humic acid decreased vase life of cut flowers of Rosa hybrida cv. White Naomi and reducing uptake solution rate by flowers. The treatment of gamma (400 Gy) + $\mathrm{CaCl}_{2}+\mathrm{HQ}+$ suc. was the best treatment for cv. Rosalin in the two seasons (Table, 2) The beneficial effect of vase solutions on irradiated cut Dendrobium flowers was to reduced ethylene production (Uthairatanakij et al., 2005).

\section{Water loss:}

Termination of vase life for cut flowers is characterized by wilting associated with an imbalance developing between water uptake through xylem conduits in stems and water loss through stomata and other structures on leaves and other organs. To better understand the onset of adverse postharvest water relations, cut flower researchers seek to acquire data on rates of water uptake and water loss. These indices are usually monitored by weighing stems and vases daily or thereabout with a single analytical balance (He et al., 2006; Lü et al., 2009; Macnish et al., 2008).

Gamma (200 Gy) gave the least rate of water loss in cv. Rosalin in the two season and priority to the control Mohammad et al. (2009) attributed the beneficial effect of sugar in vase solutions that, sugars contribute to the osmotic potential of tissues and maintain the respiration rate. Table (3) cleared that, in cv. Express HA was the perfect treatment in this concern .Coefficient with gamma (200 Gy) on cut flowers of gerbera was the fewest in perishing water compared with other treatments, supervened with gamma (400 Gy). These results are in harmony with those reported by Uthairatanakij et al. (2005) the beneficial effect of vase solutions in irradiated flowers to reduced respiration rate in cut flowers of Dendrobium.

\section{Relative fresh weight:}

During the entire period of observation, the relative fresh weight of cut flower was found to be maximum in vase solution containing oysters $(1.5 \mathrm{~g} / \mathrm{l})$ in both seasons (97.99-97.33\% respectively) in cv. Rosalin while control was (68.22-60.21\% respectively), and treatment with humic acid gave great result too with all concentrations, where with the low concentration of humic gave (94.76 - 94.48\%, respectively) and with the high concentration (95.76 - 95.22\%, respectively). These results are in accordance with those reported by Khenizy et al. (2013) on gerbera who found that humic acid at 25 and $50 \mathrm{ml} / \mathrm{l}$ and solution (A) [silver nitrate $(150 \mathrm{mg} / \mathrm{l})+$ salicylic acid $(150 \mathrm{mg} / \mathrm{l})+$ sucrose $(2 \%)+8$-hydroxyquinoline citrate (8-HQC $200 \mathrm{mg} / \mathrm{l})]$ had a superior effect on flowers fresh weight percentage. All treatments were higher than control in $\mathrm{cV}$. Rosalin in the two seasons including $\mathrm{CaCl}_{2}$ (3 g/l), similarly in gerbera (Ibrahim et al., 2011), found that, the chemical solutions of 8 -HQS at 100 or $200 \mathrm{ppm}$ or $\mathrm{CaCl}_{2}$ at 1000 or $2000 \mathrm{ppm}$ each supplemented with $4 \%$ sucrose lowered the flower weight loss. Treating with oysters $(1.5 \mathrm{~g} / \mathrm{l})$ gained an evident increment comparing to control which was the lowest followed by HA (150 $\mathrm{g} / \mathrm{l}$ ) in the two seasons. 
Table 3. Effect of holding solution treatments on water loss of Gerbera jamesonii, cv. Rosalin and Express flower heads (g/flower head) during the vase life period of 2012 and 2013 seasons.

\begin{tabular}{|c|c|c|c|}
\hline Treatments & Rosalin & Express & Mean \\
\hline \multicolumn{4}{|c|}{ First season: 2012} \\
\hline D.W. (Control) & 56.61a-f & $53.82 b-f$ & $55.22 \mathrm{a}$ \\
\hline $\mathrm{CaCl}_{2} 1 \mathrm{~g} / \mathrm{l}+\mathrm{HQ} 0.2 \mathrm{~g} / \mathrm{l}+$ suc. $20 \mathrm{~g} / \mathrm{l}$ & 62.29ab & 54.68a-f & 58.49a \\
\hline $\mathrm{CaCl}_{2} 3 \mathrm{~g} / \mathrm{l}+\mathrm{HQ} 0.2 \mathrm{~g} / \mathrm{l}+$ suc. $20 \mathrm{~g} / \mathrm{l}$ & 64.11a & 50.39c-h & $57.25 a$ \\
\hline Egg shell 0.5 g/l + HQ 0.2 g/l + suc. 20 g/l & 59.22a-d & $52.93 b-f$ & $56.08 \mathrm{a}$ \\
\hline Egg shell 1.5 g/l + HQ 0.2 g/l + suc. 20 g/l & 57.23a-e & 49.76c-h & $53.50 \mathrm{ab}$ \\
\hline Ca claw 1 g/l + HQ 0.2 g/l + suc. 20 g/l & 48.12e-h & $46.82 \mathrm{f}-\mathrm{h}$ & $47.47 \mathrm{bc}$ \\
\hline Ca claw 3 g/l + HQ 0.2 g/l + suc. 20 g/l & 59.39a-c & $52.29 b-g$ & $55.84 a$ \\
\hline Oysters 0.5 g/l + HQ 0.2 g/l + suc. 20 g/l & $53.16 b-f$ & $31.38 \mathrm{kl}$ & $42.27 \mathrm{~cd}$ \\
\hline Oysters 1.5 g/l + HQ 0.2 g/l + suc. 20 g/l & $61.92 \mathrm{ab}$ & $40.68 \mathrm{~h}-\mathrm{k}$ & $51.30 \mathrm{ab}$ \\
\hline Humic acid 50 g/l + $\mathrm{CaCl}_{2} 1 \mathrm{~g} / \mathrm{l}+$ suc. $20 \mathrm{~g} / \mathrm{l}$ & 48.28e-h & 27.131 & $37.71 d$ \\
\hline Humic acid 150 g/l + $\mathrm{CaCl}_{2} 1 \mathrm{~g} / \mathrm{l}+$ suc. $20 \mathrm{~g} / \mathrm{l}$ & $50.82 \mathrm{c}-\mathrm{h}$ & $31.08 \mathrm{kl}$ & $40.95 \mathrm{~cd}$ \\
\hline Gamma $100 \mathrm{~Gy}+\mathrm{CaCl}_{2} 1 \mathrm{~g} / \mathrm{l}+\mathrm{HQ} 0.2 \mathrm{~g} / \mathrm{l}+$ suc. $20 \mathrm{~g} / \mathrm{l}$ & 49.05d-h & $34.80 \mathrm{j}-1$ & 41.93cd \\
\hline Gamma $200 \mathrm{~Gy}+\mathrm{CaCl}_{2} 1 \mathrm{~g} / \mathrm{l}+\mathrm{HQ} 0.2 \mathrm{~g} / \mathrm{l}+$ suc. $20 \mathrm{~g} / \mathrm{l}$ & $42.05 g-j$ & $35.60 j-1$ & 38.83d \\
\hline Gamma $400 \mathrm{~Gy}+\mathrm{CaCl}_{2} 1 \mathrm{~g} / \mathrm{l}+\mathrm{HQ} 0.2 \mathrm{~g} / \mathrm{l}+$ suc. $20 \mathrm{~g} / \mathrm{l}$ & $46.36 f-i$ & 36.38i-l & 41.37 \\
\hline Mean & 54.19a & $42.70 \mathrm{~b}$ & \\
\hline
\end{tabular}

Second season: 2013

D.W. (Control)

$\mathrm{CaCl}_{2} 1 \mathrm{~g} / \mathrm{l}+\mathrm{HQ} 0.2 \mathrm{~g} / \mathrm{l}+$ suc. $20 \mathrm{~g} / \mathrm{l}$

$\mathrm{CaCl}_{2} 3 \mathrm{~g} / \mathrm{l}+\mathrm{HQ} 0.2 \mathrm{~g} / \mathrm{l}+$ suc. $20 \mathrm{~g} / \mathrm{l}$

Egg shell 0.5 g/l + HQ 0.2 g/l + suc. 20 g/l

Egg shell 1.5 g/l + HQ 0.2 g/l + suc. 20 g/l

Ca claw 1 g/l + HQ 0.2 g/l + suc. 20 g/l

Ca claw 3 g/l + HQ 0.2 g/l + suc. 20 g/l

Oysters 0.5 g/l + HQ 0.2 g/l + suc. 20 g/l

Oysters 1.5 g/l + HQ 0.2 g/l + suc. 20 g/l

Humic acid 50 g/l + $\mathrm{CaCl}_{2} 1 \mathrm{~g} / \mathrm{l}+$ suc. $20 \mathrm{~g} / \mathrm{l}$

Humic acid 150 g/l $+\mathrm{CaCl}_{2} 1 \mathrm{~g} / \mathrm{l}+$ suc. $20 \mathrm{~g} / \mathrm{l}$

Gamma $100 \mathrm{~Gy}+\mathrm{CaCl}_{2} 1$ g/l + HQ 0.2 g/l + suc. 20 g/l

Gamma $200 \mathrm{~Gy}+\mathrm{CaCl}_{2} 1$ g/l + HQ 0.2 g/l + suc. 20 g/l

Gamma $400 \mathrm{~Gy}+\mathrm{CaCl}_{2} 1 \mathrm{~g} / \mathrm{l}+\mathrm{HQ} 0.2 \mathrm{~g} / \mathrm{l}+$ suc. 20 g/l

Mean
$69.63 \mathrm{~b}$

$61.72 \mathrm{c}$

$68.2 \mathrm{~b}$

$57.63 \mathrm{e}$

$56.24 \mathrm{ef}$

$44.96 \mathrm{~m}$

$60.24 \mathrm{~cd}$

53.39g

77.59a

$45.801 \mathrm{~m}$

51.41h-j

52.75g-i

$40.66 n$

50.38j

$56.46 \mathrm{a}$

\section{$55.58 f$}

$55.21 \mathrm{f}$

59.84d

$59.74 d$

51.28ij

$47.90 \mathrm{k}$

52.93gh

$27.15 q$

$50.31 \mathrm{j}$

34.080

51.40h-j

$47.11 \mathrm{kl}$

29.71p

$25.68 \mathrm{q}$

46.28b 62.61b

$58.47 \mathrm{c}$

63.93a

$58.69 c$

$53.76 \mathrm{e}$

46.43h

56.59d

$40.27 \mathrm{i}$

$63.95 a$

39.94i

$51.41 \mathrm{f}$

49.93g

$35.19 \mathrm{k}$

38.03j

Means having the same letter are not significantly differed at .05 level of probability. 
Table 4. Effect of holding solution treatments on relative fresh weight of Gerbera jamesonii, cv. Rosalin and Express flower heads (\%) during the vase life period of 2012 and 2013 seasons.

\begin{tabular}{|c|c|c|c|}
\hline Treatments & Rosalin & Express & Mean \\
\hline \multicolumn{4}{|c|}{ First season: 2012} \\
\hline D.W. (Control) & $68.22 \mathrm{k}$ & $80.00 \mathrm{~g}-\mathrm{i}$ & $74.11 \mathrm{f}$ \\
\hline $\mathrm{CaCl}_{2} 1 \mathrm{~g} / \mathrm{l}+\mathrm{HQ} 0.2 \mathrm{~g} / \mathrm{l}+$ suc. $20 \mathrm{~g} / \mathrm{l}$ & $80.62 f-i$ & 90.45a-e & $85.54 c-e$ \\
\hline $\mathrm{CaCl}_{2} 3 \mathrm{~g} / \mathrm{l}+\mathrm{HQ} 0.2 \mathrm{~g} / \mathrm{l}+$ suc. $20 \mathrm{~g} / \mathrm{l}$ & 92.19a-e & 84.73d-h & $88.46 b-c$ \\
\hline Egg shell 0.5 g/l + HQ 0.2 g/l + suc. 20 g/l & 89.63a-f & $88.84 b-g$ & $89.24 a-d$ \\
\hline Egg shell 1.5 g/l + HQ 0.2 g/l + suc. 20 g/l & 77.91hi & $88.12 \mathrm{c}-\mathrm{g}$ & 83.02de \\
\hline Ca claw 1 g/l + HQ 0.2 g/l + suc. 20 g/l & 85.33d-h & $84.20 \mathrm{e}-\mathrm{h}$ & $84.77 \mathrm{c}-\mathrm{e}$ \\
\hline Ca claw 3 g/l + HQ 0.2 g/l + suc. 20 g/l & 93.61a-d & $88.17 \mathrm{c}-\mathrm{g}$ & 90.89a-c \\
\hline Oysters 0.5 g/l + HQ 0.2 g/l + suc. 20 g/l & 68.62jk & $77.70 h-j$ & $73.16 f$ \\
\hline Oysters 1.5 g/l + HQ 0.2 g/l + suc. 20 g/l & 97.99ab & 92.35a-e & $95.17 \mathrm{a}$ \\
\hline Humic acid 50 g/l $+\mathrm{CaCl}_{2} 1 \mathrm{~g} / \mathrm{l}+$ suc. $20 \mathrm{~g} / \mathrm{l}$ & $94.76 a-c$ & 83.12e-h & 88.94a-d \\
\hline Humic acid $150 \mathrm{~g} / \mathrm{l}+\mathrm{CaCl}_{2} 1 \mathrm{~g} / \mathrm{l}+$ suc. $20 \mathrm{~g} / \mathrm{l}$ & $95.41 \mathrm{a}-\mathrm{c}$ & 71.97i-k & 83.69de \\
\hline Gamma $100 \mathrm{~Gy}+\mathrm{CaCl}_{2} 1 \mathrm{~g} / \mathrm{l}+\mathrm{HQ} 0.2 \mathrm{~g} / \mathrm{l}+$ suc. $20 \mathrm{~g} / \mathrm{l}$ & 79.99g-i & 78.79hi & 79.39ef \\
\hline Gamma $200 \mathrm{~Gy}+\mathrm{CaCl}_{2} 1 \mathrm{~g} / \mathrm{l}+\mathrm{HQ} 0.2 \mathrm{~g} / \mathrm{l}+$ suc. $20 \mathrm{~g} / \mathrm{l}$ & 90.14a-e & 98.79a & 94.43ab \\
\hline Gamma $400 \mathrm{~Gy}+\mathrm{CaCl}_{2} 1 \mathrm{~g} / \mathrm{l}+\mathrm{HQ} 0.2 \mathrm{~g} / \mathrm{l}+$ suc. $20 \mathrm{~g} / \mathrm{l}$ & 83.96e-h & 97.73ab & $90.85 a-c$ \\
\hline Mean & $85.60 \mathrm{a}$ & $86.07 a$ & \\
\hline \multicolumn{4}{|c|}{ Second season: 2013} \\
\hline D.W. (Control) & $60.21 p$ & 78.78ij & $69.50 f$ \\
\hline $\mathrm{CaCl}_{2} 1 \mathrm{~g} / \mathrm{l}+\mathrm{HQ} 0.2 \mathrm{~g} / \mathrm{l}+$ suc. $20 \mathrm{~g} / \mathrm{l}$ & $76.00 \mathrm{kl}$ & 87.82e & 81.91d \\
\hline $\mathrm{CaCl}_{2} 3 \mathrm{~g} / \mathrm{l}+\mathrm{HQ} 0.2 \mathrm{~g} / \mathrm{l}+$ suc. $20 \mathrm{~g} / \mathrm{l}$ & 78.12i-k & 81.94gh & $80.03 e$ \\
\hline Egg shell 0.5 g/l + HQ 0.2 g/l + suc. 20 g/l & $87.24 \mathrm{e}$ & 83.88fg & $85.56 \mathrm{e}$ \\
\hline Egg shell 1.5 g/l + HQ 0.2 g/l + suc. 20 g/l & 75.30lm & $87.70 \mathrm{e}$ & 81.50de \\
\hline Ca claw 1 g/l + HQ 0.2 g/l + suc. 20 g/l & $84.42 \mathrm{f}$ & $76.82 \mathrm{j}-\mathrm{l}$ & 80.62de \\
\hline Ca claw 3 g/l + HQ 0.2 g/l + suc. 20 g/l & $92.18 d$ & $77.80 \mathrm{i}-\mathrm{k}$ & 84.99c \\
\hline Oysters 0.5 g/l + HQ 0.2 g/l + suc. 20 g/l & 65.300 & 70.20n & $67.75 \mathrm{~g}$ \\
\hline Oysters 1.5 g/l + HQ 0.2 g/l + suc. 20 g/l & 97.33ab & $86.78 \mathrm{e}$ & 92.06a \\
\hline Humic acid $50 \mathrm{~g} / \mathrm{l}+\mathrm{CaCl}_{2} 1 \mathrm{~g} / \mathrm{l}+$ suc. $20 \mathrm{~g} / \mathrm{l}$ & $94.48 \mathrm{c}$ & 79.86hi & 87.17b \\
\hline Humic acid 150 g/l + $\mathrm{CaCl}_{2} 1 \mathrm{~g} / \mathrm{l}+$ suc. 20 g/l & 95.22bc & 65.020 & $80.12 \mathrm{e}$ \\
\hline Gamma $100 \mathrm{~Gy}+\mathrm{CaCl}_{2} 1 \mathrm{~g} / \mathrm{l}+\mathrm{HQ} 0.2 \mathrm{~g} / \mathrm{l}+$ suc. $20 \mathrm{~g} / \mathrm{l}$ & $73.56 \mathrm{~m}$ & $68.36 n$ & $70.96 f$ \\
\hline Gamma $200 \mathrm{~Gy}+\mathrm{CaCl}_{2} 1 \mathrm{~g} / \mathrm{l}+\mathrm{HQ} 0.2 \mathrm{~g} / \mathrm{l}+$ suc. $20 \mathrm{~g} / \mathrm{l}$ & $82.62 \mathrm{fg}$ & $98.52 \mathrm{a}$ & $90.57 a$ \\
\hline Gamma $400 \mathrm{~Gy}+\mathrm{CaCl}_{2} 1 \mathrm{~g} / \mathrm{l}+\mathrm{HQ} 0.2 \mathrm{~g} / \mathrm{l}+$ suc. $20 \mathrm{~g} / \mathrm{l}$ & 75.31lm & 96.20bc & $85.76 \mathrm{bc}$ \\
\hline Mean & 81.23a & 81.41a & \\
\hline
\end{tabular}

Means having the same letter are not significantly differed at .05 level of probability. 
In Express cultivar, the treatment which gave the highest value was gamma (200 Gy, $400 \mathrm{~Gy}$ ) in rest of the transactions of irradiation in the two seasons. However, HA at $150 \mathrm{~g} / \mathrm{l}$ gave minimum results in each of seasons under this study. Ansari et al. (2011), found that on gerbera cut flowers humic acid at $50 \mathrm{mg} \mathrm{l}^{-1}+$ sucrose $4 \%+$ GA $2.5 \mathrm{mg} \mathrm{l}^{-1}$ gave the best fresh weight, while humic acid $50 \mathrm{mg} \mathrm{l}^{-1}+\mathrm{GA} 2.5 \mathrm{mg} \mathrm{l}^{-1}$ had the least relative fresh weight. Also, Dineshbabu et al. (2002) reported that holding solutions containing 8-HQS + sucrose extended the vase life of Dendrobium flowers and improved flower quality, as suggested by improved fresh weight. El-Saka (2002), on gerbera flowers cv. "North Star" mentioned that preservative vase solutions recorded higher rate daily fresh weight which increased as percentage. The flower weight expressed the flower freshness, flower longevity and flower senescence.

All treatments appeared arising in values over control in cv. Rosalin in both seasons. While when we compare between the two cultivars under study in terms of better treatment, we find that the transaction with oysters (1.5 g/l) was the superiority (95.17$92.06 \%$ ) in both seasons followed by gamma (200 Gy) (94.47-90.57\%) over control (74.11-69.50\%). In this respect, Hayashi et al. (1998), mentioned that, gerbera was tolerant to electron beams at 400-600 Gy.

\section{Dry weight:}

Data concerning flower dry weight (\%) as affected by gamma irradiation at the dose of $200 \mathrm{~Gy}$ plus $\mathrm{CaCl}_{2}$ at $1 \mathrm{~g} / \mathrm{l}+$ sucrose at 20 g/l (Table, 5) significant showed increased dry weight of the two cultivars Rosalin and Express followed by claw (1 g/l) where gave maximum results in both seasons. This agreed with the results found by Ibrahim, et al. (2011) who reported that, the chemical solutions of 8-HQS at 100 or $200 \mathrm{ppm}$ or $\mathrm{CaCl}_{2}$ at 1000 or $2000 \mathrm{ppm}$ each supplemented with $4 \%$ sucrose increased dry weight percentage in cut flower of gerbera.

\section{Diameter of flower head:}

The flower diameter is a suitable index to flower opening and the stem diameter is important factor of flower quality and play important role in flower marketing. All treatments in this study produce higher rates compared with control but gamma 200 Gy and humic acid $150 \mathrm{~g} / \mathrm{l}$ recorded the highest diameter in the two seasons of both cultivars. Similar results were achieved by Khenizy et al. (2013) on gerbera cut flowers the combined treatments of solution (A) [silver nitrate $(150 \mathrm{mg} / \mathrm{l})+$ salicylic acid $(150 \mathrm{mg} / \mathrm{l})$ + sucrose $(2 \%)+8$-hydroxyquinoline citrate (8 HQC $200 \mathrm{mg} / \mathrm{l}$ ) plus humic acid at 25 or $50 \mathrm{ml} / \mathrm{l}$ had a superior effect on extending life of flowers and increasing flower diameter $(\mathrm{cm})$.

In cv. Express head flowers placement in holding solution containing Ca claw ( $1 \mathrm{~g} / \mathrm{l})$ plus HQ at $0.2 \mathrm{~g} / \mathrm{l}$ and $20 \mathrm{~g} / \mathrm{l}$ sucrose enhanced the flower diameter significantly in comparison with the other interactions in both seasons. On the other hand, flower head diameter $(\mathrm{cm})$ of gerbera cv. Rosalin (Table, 6) appeared that holding flower in the holding solutions, HA (150 g/l) $+\mathrm{CaCl}_{2}$ (1 $\mathrm{g} / \mathrm{l})+$ sug. $(20 \mathrm{~g} / \mathrm{l})$ and gamma irradiation at the doses of 200 Gy plus $\mathrm{CaCl}_{2} 1 \mathrm{~g} / \mathrm{l}$ plus HQ at $0.2 \mathrm{~g}$ and $20 \mathrm{~g} / \mathrm{l}$ sucrose caused increment in the flower head diameter to $12.50,13.0$ $\mathrm{cm}$ comparing with $10.50 \& 10.50 \mathrm{~cm}$ compared with control (distilled water) in both seasons.

Data presented in the same Table (6) reflect the interaction between holding solution and varieties treatments on diameter of Gerbera jamesonii, cv. Rosalin and cv. Express flower heads during the vase life period of 2012 and 2013 seasons. It appeared that $\mathrm{cv}$. Rosalin gave maximum diameter in the two seasons comparing to the other cultivar.

\section{Chemical composition of stem and petal of Gerbera jamesonii:}

Data presented in Table (7) clear the effect of $\mathrm{CaCl}_{2}$, egg shell, Ca claw, oysters, 
Table 5. Effect of holding solution treatments on dry weight of Gerbera jamesonii, cv. Rosalin and Express flower heads (\%) during the vase life period of 2012 and 2013 seasons.

\begin{tabular}{|c|c|c|c|}
\hline Treatments & Rosalin & Express & Mean \\
\hline \multicolumn{4}{|c|}{ First season: 2012} \\
\hline D.W. (Control) & 20.03i-k & 25.89e-h & 22.96ef \\
\hline $\mathrm{CaCl}_{2} 1 \mathrm{~g} / \mathrm{l}+\mathrm{HQ} 0.2 \mathrm{~g} / \mathrm{l}+$ suc. $20 \mathrm{~g} / \mathrm{l}$ & 28.30c-g & 18.19jk & 23.25ef \\
\hline $\mathrm{CaCl}_{2} 3 \mathrm{~g} / \mathrm{l}+\mathrm{HQ} 0.2 \mathrm{~g} / \mathrm{l}+$ suc. $20 \mathrm{~g} / \mathrm{l}$ & $16.96 \mathrm{k}$ & 17.55 & $17.26 \mathrm{~g}$ \\
\hline Egg shell 0.5 g/l + HQ 0.2 g/l + suc. 20 g/l & $30.25 c-e$ & $28.80 \mathrm{c}-\mathrm{g}$ & 29.53bc \\
\hline Egg shell 1.5 g/l + HQ 0.2 g/l + suc. 20 g/l & $29.85 c-f$ & $28.94 c-g$ & 29.40bc \\
\hline Ca claw 1 g/l + HQ 0.2 g/l + suc. 20 g/l & $39.32 \mathrm{a}$ & 28.93c-g & 34.13a \\
\hline Ca claw 3 g/l + HQ 0.2 g/l + suc. 20 g/l & 27.16c-h & 26.71d-h & $26.94 \mathrm{~cd}$ \\
\hline Oysters 0.5 g/l + HQ 0.2 g/l + suc. 20 g/l & 31.18b-d & $30.75 c-e$ & 30.97ab \\
\hline Oysters 1.5 g/l + HQ 0.2 g/l + suc. 20 g/l & 22.61h-j & $28.69 c-g$ & 25.65de \\
\hline Humic acid 50 g/l + $\mathrm{CaCl}_{2} 1 \mathrm{~g} / \mathrm{l}+$ suc. $20 \mathrm{~g} / \mathrm{l}$ & 20.69i-k & $24.40 g-\mathrm{i}$ & 22.55ef \\
\hline Humic acid $150 \mathrm{~g} / \mathrm{l}+\mathrm{CaCl}_{2} 1 \mathrm{~g} / \mathrm{l}+$ suc. $20 \mathrm{~g} / \mathrm{l}$ & $18.81 \mathrm{jk}$ & 22.35h-j & $20.58 f g$ \\
\hline Gamma $100 \mathrm{~Gy}+\mathrm{CaCl}_{2} 1 \mathrm{~g} / \mathrm{l}+\mathrm{HQ} 0.2 \mathrm{~g} / \mathrm{l}+$ suc. $20 \mathrm{~g} / \mathrm{l}$ & 24.20g-i & 25.01f-i & 24.61de \\
\hline Gamma $200 \mathrm{~Gy}+\mathrm{CaCl}_{2} 1 \mathrm{~g} / \mathrm{l}+\mathrm{HQ} 0.2 \mathrm{~g} / \mathrm{l}+$ suc. $20 \mathrm{~g} / \mathrm{l}$ & $32.23 b c$ & 36.28ab & 34.26a \\
\hline Gamma $400 \mathrm{~Gy}+\mathrm{CaCl}_{2} 1 \mathrm{~g} / \mathrm{l}+\mathrm{HQ} 0.2 \mathrm{~g} / \mathrm{l}+$ suc. $20 \mathrm{~g} / \mathrm{l}$ & 26.96d-h & $28.68 \mathrm{c}-\mathrm{g}$ & $27.82 b-c$ \\
\hline Mean & 26.33a & 26.51a & \\
\hline \multicolumn{4}{|c|}{ Second season: 2013} \\
\hline D.W. (Control) & 19.96j-l & $28.14 \mathrm{e}-\mathrm{g}$ & 24.05e \\
\hline $\mathrm{CaCl}_{2} 1 \mathrm{~g} / \mathrm{l}+\mathrm{HQ} 0.2 \mathrm{~g} / \mathrm{l}+$ suc. $20 \mathrm{~g} / \mathrm{l}$ & 30.30d-f & $18.53 \mathrm{kl}$ & $24.42 \mathrm{e}$ \\
\hline $\mathrm{CaCl}_{2} 3 \mathrm{~g} / \mathrm{l}+\mathrm{HQ} 0.2 \mathrm{~g} / \mathrm{l}+$ suc. $20 \mathrm{~g} / \mathrm{l}$ & 17.241 & 17.561 & $17.40 \mathrm{~g}$ \\
\hline Egg shell 0.5 g/l + HQ 0.2 g/l + suc. 20 g/l & 29.79ef & 30.85de & $30.32 b-c$ \\
\hline Egg shell 1.5 g/l + HQ 0.2 g/l + suc. 20 g/l & 34.73cd & 30.80de & 32.76bc \\
\hline Ca claw 1 g/l + HQ 0.2 g/l + suc. 20 g/l & 39.20bc & $28.20 \mathrm{e}-\mathrm{g}$ & 33.70b \\
\hline Ca claw 3 g/l + HQ 0.2 g/l + suc. 20 g/l & 31.12de & 27.92e-h & 29.52cd \\
\hline Oysters 0.5 g/l + HQ 0.2 g/l + suc. 20 g/l & $19.15 \mathrm{j}-\mathrm{l}$ & 30.31d-f & 24.73e \\
\hline Oysters 1.5 g/l + HQ 0.2 g/l + suc. 20 g/l & 23.20h-k & 27.47e-h & 25.33e \\
\hline Humic acid $50 \mathrm{~g} / \mathrm{l}+\mathrm{CaCl}_{2} 1 \mathrm{~g} / \mathrm{l}+$ suc. $20 \mathrm{~g} / \mathrm{l}$ & 19.38j-l & 25.95f-i & 22.67ef \\
\hline Humic acid 150 g/l + $\mathrm{CaCl}_{2} 1 \mathrm{~g} / \mathrm{l}+$ suc. $20 \mathrm{~g} / \mathrm{l}$ & $19.51 \mathrm{j}-\mathrm{l}$ & 20.00j-l & $19.76 f g$ \\
\hline Gamma $100 \mathrm{~Gy}+\mathrm{CaCl}_{2} 1 \mathrm{~g} / \mathrm{l}+\mathrm{HQ} 0.2 \mathrm{~g} / \mathrm{l}+$ suc. $20 \mathrm{~g} / \mathrm{l}$ & $23.41 \mathrm{~h}-\mathrm{j}$ & 22.08i-1 & 22.74ef \\
\hline Gamma $200 \mathrm{~Gy}+\mathrm{CaCl}_{2} 1 \mathrm{~g} / \mathrm{l}+\mathrm{HQ} 0.2 \mathrm{~g} / \mathrm{l}+$ suc. $20 \mathrm{~g} / \mathrm{l}$ & 48.57a & 40.11b & $44.34 \mathrm{a}$ \\
\hline Gamma $400 \mathrm{~Gy}+\mathrm{CaCl}_{2} 1$ g/l + HQ 0.2 g/l + suc. 20 g/l & 29.94d-f & 28.00e-h & 28.97d \\
\hline Mean & $27.54 a$ & $26.85 a$ & \\
\hline
\end{tabular}

Means having the same letter are not significantly differed at .05 level of probability. 
Table 6. Effect of holding solution treatments on diameter of Gerbera jamesonii, cv. Rosalin and Express flower heads (cm) during the vase life period of 2012 and 2013 seasons.

\begin{tabular}{|c|c|c|c|}
\hline Treatments & Rosalin & Express & Mean \\
\hline \multicolumn{4}{|c|}{ First season: 2012} \\
\hline D.W. (Control) & 10.50g-i & $10.00 \mathrm{i}-\mathrm{k}$ & $10.25 f$ \\
\hline $\mathrm{CaCl}_{2} 1 \mathrm{~g} / \mathrm{l}+\mathrm{HQ} 0.2 \mathrm{~g} / \mathrm{l}+$ suc. $20 \mathrm{~g} / \mathrm{l}$ & $11.50 \mathrm{~d}-\mathrm{f}$ & 10.90f-h & $11.20 \mathrm{~b}-\mathrm{d}$ \\
\hline $\mathrm{CaCl}_{2} 3 \mathrm{~g} / \mathrm{l}+\mathrm{HQ} 0.2 \mathrm{~g} / \mathrm{l}+$ suc. $20 \mathrm{~g} / \mathrm{l}$ & $11.50 \mathrm{~d}-\mathrm{f}$ & $10.10 \mathrm{i}-\mathrm{k}$ & 10.80de \\
\hline Egg shell 0.5 g/l + HQ 0.2 g/l + suc. 20 g/l & $12.27 \mathrm{bc}$ & $10.25 h-j$ & $11.26 \mathrm{a}-\mathrm{d}$ \\
\hline Egg shell 1.5 g/l + HQ 0.2 g/l + suc. 20 g/l & $12.00 \mathrm{~b}-\mathrm{d}$ & $10.00 \mathrm{i}-\mathrm{k}$ & 11.00c-e \\
\hline Ca claw 1 g/l + HQ 0.2 g/l + suc. 20 g/l & $11.77 \mathrm{~cd}$ & $11.50 \mathrm{~d}-\mathrm{f}$ & 11.64ab \\
\hline Ca claw 3 g/l + HQ 0.2 g/l + suc. 20 g/l & $12.00 \mathrm{~b}-\mathrm{d}$ & $10.00 \mathrm{i}-\mathrm{k}$ & 11.00c-e \\
\hline Oysters 0.5 g/l + HQ 0.2 g/l + suc. 20 g/l & $11.67 \mathrm{c}-\mathrm{e}$ & $10.27 \mathrm{~h}-\mathrm{j}$ & 10.97de \\
\hline Oysters 1.5 g/l + HQ 0.2 g/l + suc. 20 g/l & $12.27 \mathrm{bc}$ & $10.10 \mathrm{i}-\mathrm{k}$ & $11.19 b-d$ \\
\hline Humic acid $50 \mathrm{~g} / \mathrm{l}+\mathrm{CaCl}_{2} 1 \mathrm{~g} / \mathrm{l}+$ suc. $20 \mathrm{~g} / \mathrm{l}$ & 12.50ab & 10.50g-i & $11.50 \mathrm{a}-\mathrm{c}$ \\
\hline Humic acid $150 \mathrm{~g} / \mathrm{l}+\mathrm{CaCl}_{2} 1 \mathrm{~g} / \mathrm{l}+$ suc. $20 \mathrm{~g} / \mathrm{l}$ & 13.00a & 10.50g-i & $11.75 a$ \\
\hline Gamma $100 \mathrm{~Gy}+\mathrm{CaCl}_{2} 1 \mathrm{~g} / \mathrm{l}+\mathrm{HQ} 0.2 \mathrm{~g} / \mathrm{l}+$ suc. $20 \mathrm{~g} / \mathrm{l}$ & $12.00 \mathrm{~b}-\mathrm{d}$ & $9.77 \mathrm{jk}$ & 10.89de \\
\hline Gamma $200 \mathrm{~Gy}+\mathrm{CaCl}_{2} 1 \mathrm{~g} / \mathrm{l}+\mathrm{HQ} 0.2 \mathrm{~g} / \mathrm{l}+$ suc. $20 \mathrm{~g} / \mathrm{l}$ & $12.50 \mathrm{ab}$ & $11.00 \mathrm{e}-\mathrm{g}$ & $11.75 a$ \\
\hline Gamma $400 \mathrm{~Gy}+\mathrm{CaCl}_{2} 1 \mathrm{~g} / \mathrm{l}+\mathrm{HQ} 0.2 \mathrm{~g} / \mathrm{l}+$ suc. $20 \mathrm{~g} / \mathrm{l}$ & $11.77 \mathrm{~cd}$ & $9.50 \mathrm{k}$ & $10.64 \mathrm{ef}$ \\
\hline Mean & $11.95 \mathrm{a}$ & 10.31b & \\
\hline \multicolumn{4}{|c|}{ Second season: 2013} \\
\hline D.W. (Control) & $10.50 c-e$ & 10.00de & $10.25 d$ \\
\hline $\mathrm{CaCl}_{2} 1 \mathrm{~g} / \mathrm{l}+\mathrm{HQ} 0.2 \mathrm{~g} / \mathrm{l}+$ suc. $20 \mathrm{~g} / \mathrm{l}$ & $11.50 \mathrm{a}-\mathrm{d}$ & 11.00b-e & $11.25 \mathrm{a}-\mathrm{d}$ \\
\hline $\mathrm{CaCl}_{2} 3 \mathrm{~g} / \mathrm{l}+\mathrm{HQ} 0.2 \mathrm{~g} / \mathrm{l}+$ suc. $20 \mathrm{~g} / \mathrm{l}$ & $11.50 \mathrm{a}-\mathrm{d}$ & 12.20de & $10.85 a-d$ \\
\hline Egg shell 0.5 g/l + HQ 0.2 g/l + suc. 20 g/l & 12.50ab & 11.00b-e & 11.75ab \\
\hline Egg shell 1.5 g/l + HQ 0.2 g/l + suc. 20 g/l & $12.00 \mathrm{a}-\mathrm{c}$ & $9.50 \mathrm{e}$ & $10.75 b-d$ \\
\hline Ca claw 1 g/l + HQ 0.2 g/l + suc. 20 g/l & $12.00 \mathrm{a}-\mathrm{c}$ & $11.50 \mathrm{a}-\mathrm{d}$ & 11.75ab \\
\hline Ca claw 3 g/l + HQ 0.2 g/l + suc. 20 g/l & $11.50 a-d$ & 11.00b-e & $11.25 \mathrm{a}-\mathrm{d}$ \\
\hline Oysters 0.5 g/l + HQ 0.2 g/l + suc. 20 g/l & $12.00 \mathrm{a}-\mathrm{c}$ & $9.50 \mathrm{e}$ & $10.75 b-d$ \\
\hline Oysters 1.5 g/l + HQ 0.2 g/l + suc. 20 g/l & 12.50ab & 10.30de & $11.40 \mathrm{a}-\mathrm{d}$ \\
\hline Humic acid 50 g/l + $\mathrm{CaCl}_{2} 1 \mathrm{~g} / \mathrm{l}+$ suc. $20 \mathrm{~g} / \mathrm{l}$ & 12.50ab & $11.50 \mathrm{a}-\mathrm{d}$ & $12.00 \mathrm{a}$ \\
\hline Humic acid 150 g/l + $\mathrm{CaCl}_{2} 1 \mathrm{~g} / \mathrm{l}+$ suc. $20 \mathrm{~g} / \mathrm{l}$ & 13.00a & 11.00b-e & $12.00 \mathrm{a}$ \\
\hline Gamma $100 \mathrm{~Gy}+\mathrm{CaCl}_{2} 1 \mathrm{~g} / \mathrm{l}+\mathrm{HQ} 0.2 \mathrm{~g} / \mathrm{l}+$ suc. $20 \mathrm{~g} / \mathrm{l}$ & $13.00 \mathrm{a}$ & 10.00de & $11.50 \mathrm{a}-\mathrm{c}$ \\
\hline Gamma $200 \mathrm{~Gy}+\mathrm{CaCl}_{2} 1 \mathrm{~g} / \mathrm{l}+\mathrm{HQ} 0.2 \mathrm{~g} / \mathrm{l}+$ suc. $20 \mathrm{~g} / \mathrm{l}$ & $13.00 \mathrm{a}$ & 11.00b-e & $12.00 \mathrm{a}$ \\
\hline Gamma $400 \mathrm{~Gy}+\mathrm{CaCl}_{2} 1 \mathrm{~g} / \mathrm{l}+\mathrm{HQ} 0.2 \mathrm{~g} / \mathrm{l}+$ suc. $20 \mathrm{~g} / \mathrm{l}$ & $11.50 \mathrm{a}-\mathrm{d}$ & $9.50 \mathrm{e}$ & 10.50cd \\
\hline Mean & $12.07 \mathrm{a}$ & $10.50 \mathrm{~b}$ & \\
\hline
\end{tabular}

Means having the same letter are not significantly differed at .05 level of probability. 
Ola A. Amin; Azza M. Abdel-Moneim and Hanan M.A.Youssef

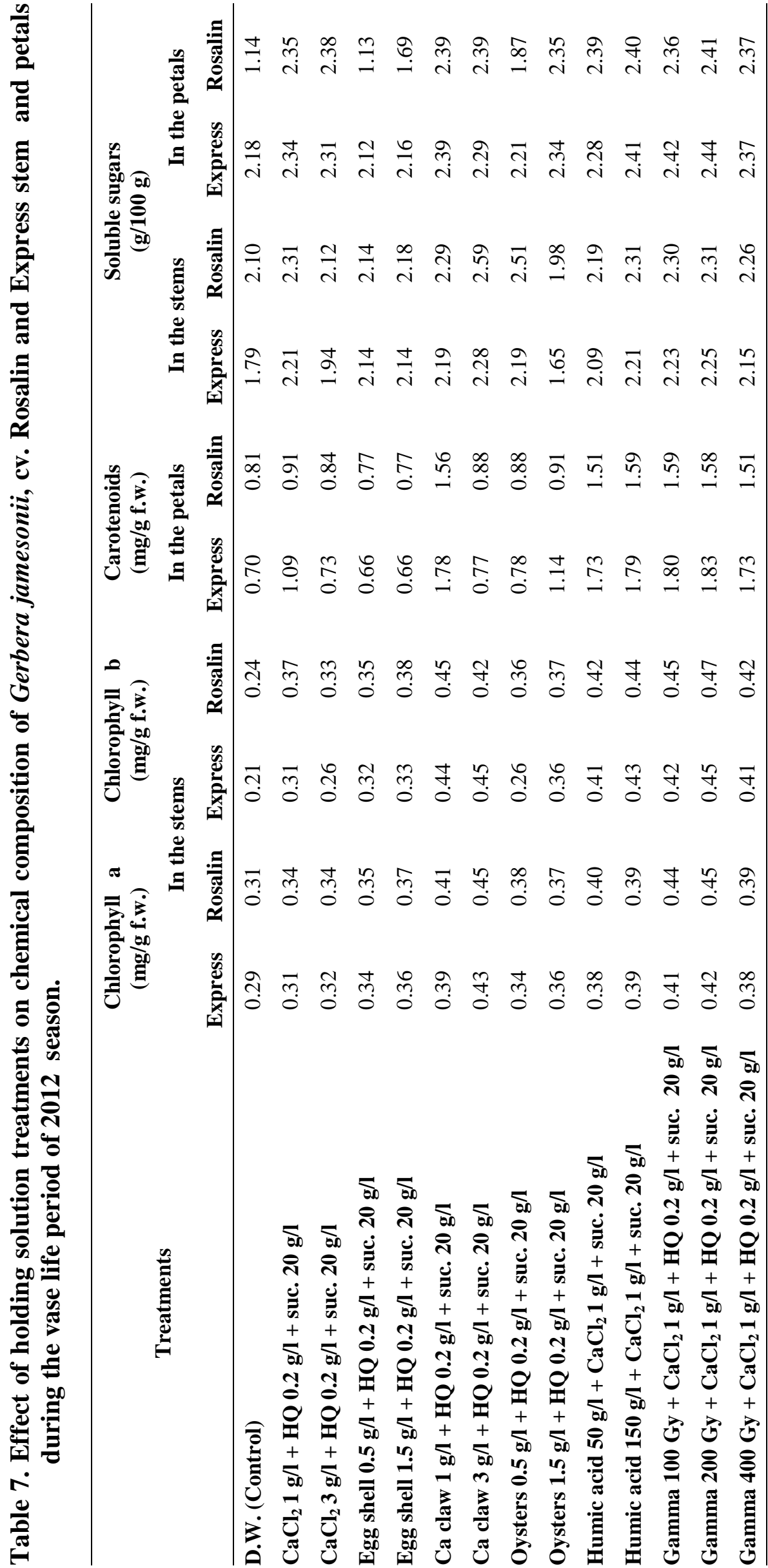


and three doses of gamma irradiation of Gerbera jamesonii, it could be concluded that chlorophyll a and b content in the stems (mg/g f.w.) was increased in response to all the used holding solutions comparing with control in the two cultivars. Treating with $\mathrm{CaCl}_{2}$ at $3 \mathrm{~g} / \mathrm{l}+\mathrm{HQ}$ at $0.2 \mathrm{~g} / \mathrm{l}$ and suc. at 20 $\mathrm{g} / \mathrm{l}$, or gamma rays at 200 or $400 \mathrm{~Gy}+$ $\mathrm{CaCl}_{2} 1 \mathrm{~g} / \mathrm{l}+\mathrm{HQ} 0.2 \mathrm{~g} / \mathrm{l}+$ sucrose at $20 \mathrm{~g} / \mathrm{l}$ registered high increments in chlorophyll a in the stems and soluble sugars in the (stems, and petals) compared to other treatments. However, chlorophyll b in the stems and carotenoids in the petals contents recorded high increases with gamma rays at $200 \mathrm{~Gy}+$ $\mathrm{CaCl}_{2} 1 \mathrm{~g} / \mathrm{l}+\mathrm{HQ} 0.2 \mathrm{~g} / \mathrm{l}+20 \mathrm{~g} / \mathrm{l}$ sucrose in both cultivars. This agreed with Tanabe and Dohino (1993) they found that, color cannot be used as a reference to indicate if the flower is tolerant to radiation. Alpinia, Curcuma, Eustoma and Gerbera present some grades of petal discoloration with damaging doses of both kinds of radiation and this symptom can be a result of direct or indirect effects of radiation on pigments. Also Ibrahim et al. (2011) observed that in cut flowers of gerbera, the chemical solutions of 8-HQS at 100 or $200 \mathrm{ppm}$ or $\mathrm{CaCl}_{2}$ at 1000 or 2000 ppm each supplemented with $4 \%$ sucrose resulted in the highest soluble sugars content in petals of the flowers.

Farahat and Gaber (2009) mentioned that, window leaf cut foliage when holding in different preservative solutions recorded the highest content of total soluble sugars with $\mathrm{CaCl}_{2}$ at the rate of $1000 \mathrm{ppm}$. However, 8-HQS 200 or $400 \mathrm{ppm}+30 \mathrm{~g} / \mathrm{l}$ sucrose recorded the lowest values.

\section{REFERENCES}

Amariutei, A.; Cracuin, C. and Burz, I. (1995). Changes in the ultrastructure of cut gerbera inflorescences during vase life. Acta Hort., 405:108-116.

Ansari S.; Hadavi, E.; Salehi, M. and Moradi, P. (2011). Application of microorganisms compared with nanoparticles of silver, humic acid and gibberellic acid on vase life of cut Gerbera Good Timming. Journal of Ornamental and Horticultural Plants, 1(1):27-33.

Bulent, A. and Senay, M. (2012). Effects of pre-harvest and postharvest calcium and modified atmosphere treatments on vase life of gerbera. J. Food, Agric. \& Environment, 10 (3\&4):968-971.

Celikel, F.G. and Karaaly, Y. (1995). Effect of postharvest factors on flower quality and longevity of cut carnation (Dianthus caryophyllus L.). Acta Hort., 405:156163.

Chen, D.S.; Li, N.H.; Wang, J.M.; Ding, Y.X. and Wang, X.J. (2004). Effect of calcium chloride on preservation of cut flowers of Gerbera hybrida. Acta Botanic Yunnanica, 26(3): 345-348.

Choudhary, M.L. and Prasad, K.V. (2000). Protected cultivation of ornamental crops-An insight. Indian Hort., 45(1):4953.

Chutichudet, B. and Chutichudet, P. (2012). Influence of calcium solutions to physiological changes of 'Chiang Mai Pink’ Patumma cut flower. International Journal of Agricultural Research, 7(3):121-133.

Cortes, M.H.; Frias, A.A.; Moreno, S.G.; Piña, M.M.; Guzmán, G.H.D.L.C. and Sandoval, S.G. (2011). The effects of calcium on postharvest water status and vase life of Rosa hybrida cv. Grand Gala., Int. J. Agric. Biol., 13: 233-238.

Daengprok, W; Issigonis, K.; Mine, Y.; Pornsinpatip, P.; Garnjanagoonchorn, W. and Naivikul, O. (2003). Chicken eggshell matrix proteins enhance calcium transport in the human intestinal epithelial cells. Caco-Journal of Agricultural and food Chemistry, 51:6056-6061.

Dineshbabu, M.; Jawaharlal, M. and Yijayakumar, M. (2002). Influence of holding solutions on the post harvest life 
of Dendrobium hybrid Sonia-17. South Indian Horticulture, 50(4/6):451-457.

Dohino, T. and Hayashi, T. (1995). Avoidance of electron irradiation induced injuries of chrysanthemum cut flowers with preservatives. Res. Bull. Pl. Prot. Jap., 31:95-100.

Dubois, M., Smith, F.; Iiles, K.A.; Hamilton, J.K. and Rebers, P.A. (1956). Colorimetric method for determination of sugars and related substances. Annal. Chem., 28(3):350-356.

Duncan, D.B. (1955). Multiple range and multiple F-tests. Biometrices, 11:1-42.

El-Saka, M.M. (2002). Physiological and biological changes in the cut Gerbera Jamesonii cv. North Star flowers under effect of ethylene inhibitors and Jasmonic acid during vase life. J. Product. \& Dev., 7(2):205-217.

Emongor, V.E. (2004). Effects of gibberellic acid on postharvest quality and vase life of Gerbera jamesonii cut flowers. ActaHort. Sinica, 31(4):408-410.

Farahat, M.M. and Gaber, A. (2009). Influence of preservative materials on postharvest performance of cut window leaf foliage (Monstera deliciosa). Acta Hort., 3: 1715-1718.

Gerasopoulos, D. and Chebli, B. (1999). Effects of pre- and postharvest calcium applications on the vase life of cut gerberas., J. Hort. Sci. Biot., 74:78-81.

Haasbroek, F.J.; Rousseau, G.G.; De Villers, J.F. (1973). Effect of gamma-rays on cut blooms of Protea compacta R.Br., P. longiflora Lamark and Leucospermum cordifolium Salis ex Knight. Agroplantae, 5: 33-42.

Hamidah, S.; Osman, M.S.; Zainon, O.; Ridzuan, L.M. (2006). Development of gamma irradiation as a quarantine treatment of mites on cut flowers. Acta Hort., 1(68):0567-7572.

Hayashi, T. and Dohino, T. (1995). Effect of the components of vase solution on the vase-life of irradiated cut chrysanthemums. Rep. Natl. Food Res. Inst., 59:17- 19.

Hayashi, T., and Todoriki, S. (1996). Sugars prevent the detrimental effects of gamma irradiation on cut chrysanthemums, Hort.Sci., 31(1):117-119.

Hayashi, T.; Kikuchi, O. and Dohino, T. (1998). Electron beam disinfestations of cut flowers and their radiation tolerance. Radiat. Phys. Chem., 51(2): 175-179.

He, S.; Joyce, D.C.; Irving, D.E. (2006). Competition for water between inflorescences and leaves in cut flowering stems of Grevillea 'Crimson Yul-lo’. J. Hort. Sci. Biotechnol., 81:891-897.

Ichimura, K.; Fujiwara, T.; Yamauchi, Y.; Horie, H. and Kohata, K. (2005). Effects of tea-seed saponins on the vase life, hydraulic conductance and transpiration of cut rose flowers. Japan Agric. Res. Quat., 39:115-119.

Ibrahim, Soad M.M.; Taha, Lobna S. and Eid, Rawia A. (2011). Extending postharvest life and keeping quality of gerbera cut-flowers using some chemical preservatives. Journal of Applied Sciences Research, 7(7):1233-1239.

Keshavarzi, L. and Chamani, E. (2011). Effects of hinokitiol, humic acid, sucrose, and silver thiosulfate (STS) as a short-time (pulsing) treatment on vase life of cut 'Yelloween' lily flowers. Iranian Journal of Horticultural Science, 42(4)393-402.

Khenizy, Soad A.M.; Zaky, Amal A. and ElShewaikh, Yasser M.E. (2013). Effect of humic acid on vase life of gerbera flowers after cutting. Journal of Horticultural Science \& Ornamental Plants, 5(2):127-136.

Kikuchi, Olivia. K.; DelMastro, N.L. and Wiendl, F.M. (1995). Preservative solution for gamma irradiated 
chrysanthemum cut flowers. Radiat. Phys. Chem., 46 (4-6):1309-1311.

Kikuchi,O. K. (2000). Orchid flowers tolerance to gamma-radiation. Radiat. Phys. Chem., 57:555-557.

Lü, P.; He, S.; Liu, J.; Cao, J.; Joyce, D.C. (2009). A new apparatus for continuous automatic measurement of water relations in stems of cut flowers. Intl. Conf. Plant Vascular Biol (Agr. 2009, Abst. Book, Beibei, China), 314-315.

Macnish, A.J.; Leonard, R.T. and Nell, T.A. (2008). Treatment with chlorine dioxide extends the vase life of selected cut flowers. Postharvest Biol. Technol., 50:197-207.

Mencarelli, F.; Agostini, R.; Botondi, R. and Massautini, R. (1995). Ethylene production, ACC content, PAL and POD activities in excised sections of straight and bent gerbera scapes. J. Hort. Sci., 70(3):409-416.

Mohammad, E.A.; Vali, R. and Sahar, B.Z. (2009). Influence of pulse chemical treatments on water relation in cut gerbera (Gerbera jamesonii cv. Pags) flowers. Journal of Food, Agriculture \& Environment, 7 (1):182-185.

Mojgan, K.; Mirzakhani, A. and Hakimi, L. (2013). Influences of Thyme oil (Thymus vulgaris L.), Aloe vera gel and some chemical substances on vase- life of cut Rosa hybrida cv. White Naomi. International Journal of Agronomy and Plant Production, 4 (5):970-975.

Moran, R. (1982). Formula for determination of chlorophyllous pigment extracted with N-N-dimethyl formamide. Plant Physiol., 69:1376-81.

Mollusks (2011). Nutrition Facts and Analysis for Mollusks, oyster, eastern, wild, raw. Nutritiondata. Com. Retrieved, 2011:08-16.

MSTAT Computer Program (1985). Software program for desigen, management and analysis experimental (version 4.0), Michigan State Univ.

Nagaraja, G.S.; Gopinath, R. and Gowda, J. V. (2000). Effect of pulsing with sucrose and silver nitrate on vase life of gerbera. Mysore J. Agric. Sci., 34(2):102-104.

Nardi, S.; Pizzeghello, D.; Muscolo, A. and Vianello, A. (2002). Physiological effects of humic substances on higher plants. Soil Biol. Biochem., 34:15271536.

Nazari, M.J.; Ahmad, K.; Mostafa, A. and Roya, K. (2011). Postharvest evaluation of vase life, stem bending and screening of cultivars of cut gerbera (Gerbera jamesonii Bolus ex. Hook f.) flowers. African Journal of Biotechnology, 10(4):560-566.

Reyes-Arribas, T.; Barrett, J.E.; Nell, T.A. and Clark, D. (2000). Effect of ethylene, sucrose and benzyl adenine on leaf senescence of two chrysanthemum cultivars "Tara" and "Boaldi". Acta Hort., 5(18):125-129.

Samaneh, B.; Hadav, I.E. and Moradi, P. (2015). Interaction effect of sucrose, salicylic acid and 8-hydroxyquinoline sulfate on vase-life of cut Gerbera flower. Current Agric. Res. J., 2(5-29):14.

Samiee, M.; Zarchini, M.; Vand, S.H. and Hashemabadi, D. (2013). Improvement vase life, protein content and postharvest quality of Dendranthema grandiflorum L. cv White by artemisia oil. Annals of Biological Research., 4 (3):127-129.

Saric, M.; Kastrori, R.; Curic, R.; Cupina, T. and Geric, I. (1967). Chlorophyll Determination. Praktikum iz Fiziologije Biljaka. Univ. u Novom Sadu, Beograd, 215.

Snedecor, C.W. and Cochran, W.G. (1972). Statistical Methods $6^{\text {th }}$ ed. Iowa Sate Univ. Press Ames, Iowa, U.S.A. 507.

Singh, U.C. and Tiwari, A.K. (2002). Effect of pulsing on postharvest life of rose cv. 


\section{Ola A. Amin; Azza M. Abdel-Moneim and Hanan M.A.Youssef}

Dorris. J. South Indian Hort., 50(1/3):140-144.

Tanabe, K. and Dohino, T. (1993). Effects of electron beam irradiation on cut flowers. Res. Bull. Plant. Prot. Japan, 29:1-9.

Tanabe, K. and Dohino, T. (1995). Responses of 17 species of cut flowers to electron beam irradiation. Res. Bull. Pl. Prot. Japan, 31:89-94.

Uthairatanakij, A.; Jansri, S.; Jitareerat, P. and Kanlayanarat, S. (2005). Effect of pre- harvest calcium spraying on gamma irradiate inflorescences of 'Walter Symposium "New Frontier of Irradiated Food and Non-Food Products" 22-23 September 2005, KMUTT, Bangkok, Thailand.

Van Doorn, W.G. and Witte, Y.D. (1994). Effect of bacteria on scape bending in cut Gerbera jamesonii flowers. J. Amer. Soc. Hort. Sci., 119:568-571.

Walton, H.V.; Cotterill, O.J. and Vandepopuliere, J.M. (1973). Composition of shell waste from egg breaking plants. Poultry Sci., 52:18361841.

\section{تأثثير التشعيع بأثتعة جاما وبعض محاليل الحفظ على فتره بقاء ازهار الجيربيرا}

$$
\begin{aligned}
& \text { علا عواد أمين، عزة محمد عبد المنعم، حنان محمد أحمد يوسف هُ هُ } \\
& \text { قسم بحوث الزينة وتتسيق الحدائق، معهد بحوث البساتين، مركز البحوث الزر اعية، الجيزة، مصر. }
\end{aligned}
$$

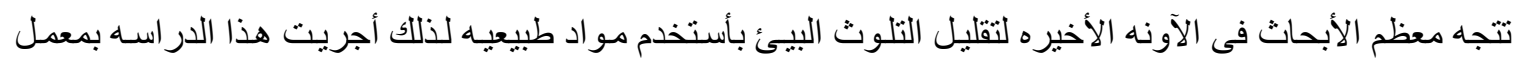

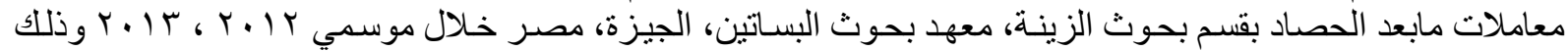

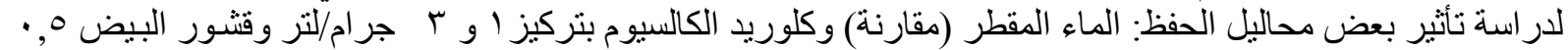

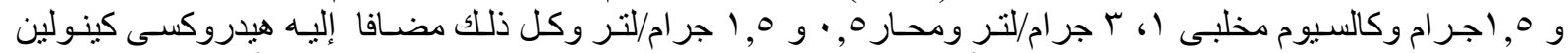

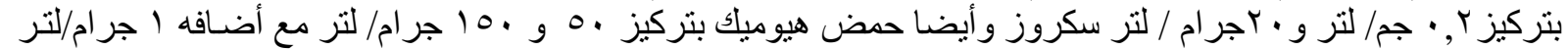

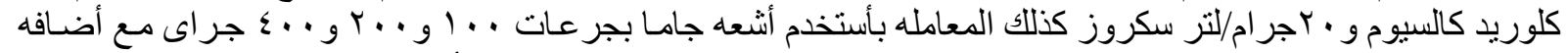

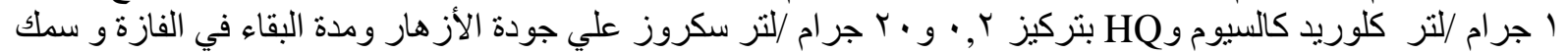

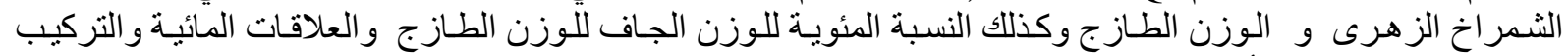

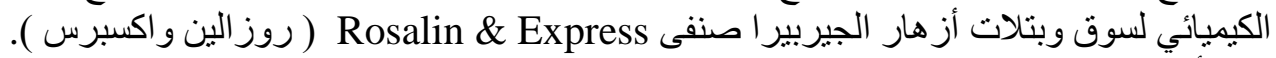

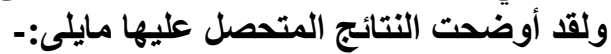

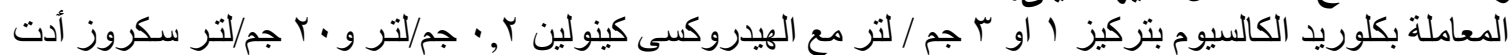

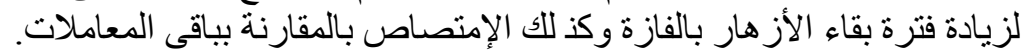

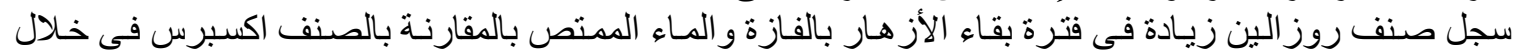
موسمى التجربة. أدت معاملة الأزهار بأثُعة جاما بمقدار .. جر اى للوصول لأقل فقد للماء من خلال أزهار صنف روز الين خـلال الموسمين.

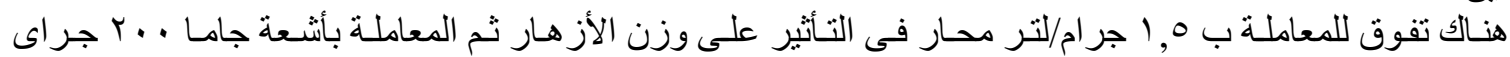

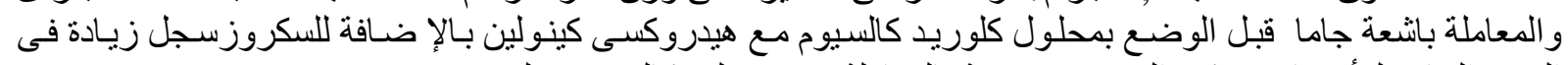

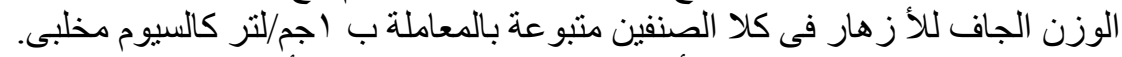

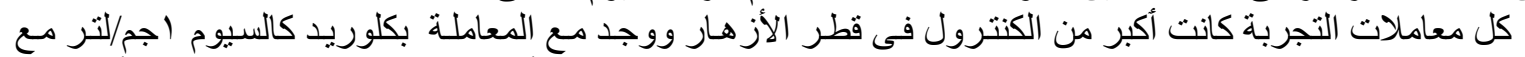

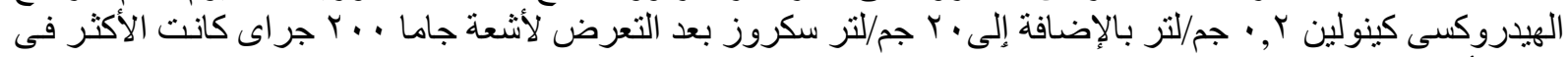

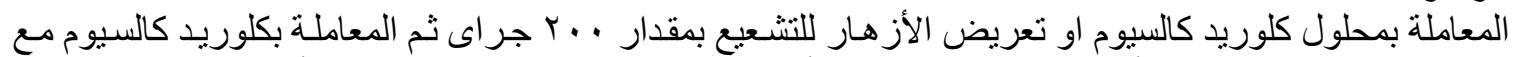
قطر الأزهار.

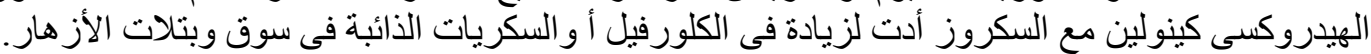

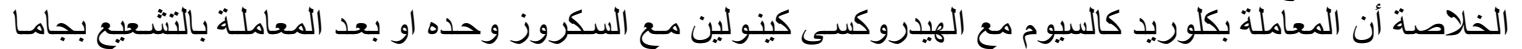

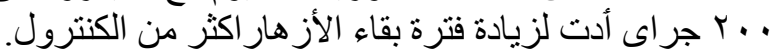



